\title{
Hetaera Esmeralda
}

Der Name „Hetaera Esmeralda“ wird zum ersten Mal im dritten Kapitel des Romans erwähnt. Er bezieht sich auf einen Schmetterling aus einem der ,farbig illustrierten Bücher über exotische Falter und Meergetier" (DF: 26) von Jonathan Leverkühn. Die Schmetterlinge werden als „Insekten [bezeichnet], die in phantastisch übertriebener Schönheit ein ephemeres Leben fristen, und von denen einige den Eingeborenen als böse Geister gelten, die die Malaria bringen“" (ebd.; Herv. A. O). Schon diese kurze Beschreibung fasst die Eigenschaften dieser Insekten zusammen: die Schönheit, das vergängliche Leben, ihre Betrachtung als dämonische Tiere, die Krankheiten hervorrufen. ,Hetaera Esmeralda', eine Art von Schmetterling ,in durchsichtiger Nacktheit den dämmernden Laubschatten liebend“ (DF: 27), hat „nur einen dunklen Farbfleck in Violett und Rosa“ (ebd.) auf ihren Flügeln, ,der sie, da man sonst nichts von ihr sieht, im Flug einem windgeführten Blütenblatt gleichen läßt" (ebd.). Eine weitere Eigenschaft von einigen Arten dieser Tiere ist, dass sie in der Lage sind, sich unsichtbar zu machen. Gerade deswegen besäßen sie etwas Schwermütiges: Astrid Roffmann vertritt in ihrer Untersuchung, die sich mit der Natur im Werk Thomas Manns befasst, die Auffassung, Schmetterlinge seien von Melancholie gekennzeichnet, weil sie an ihrer Isolation leiden. ${ }^{1}$

Die Erwähnung der im Roman genannten Eigenschaften dieses Schmetterlings bzw. anderer Schmetterlinge sowie weiterer Eigenschaften von Tieren und Naturphänomenen im dritten Kapitel findet eine Entsprechung in einer wichtigen Figur des Romans, nämlich Hetaera Esmeralda. ${ }^{2}$ Leverkühn nennt die Prostituierte im

${ }^{1}$ Siehe Roffmann, Astrid: „Keine freie Note mehr“. Natur im Werk Thomas Manns. Würzburg: Königshausen \& Neumann 2003, S. 191. Vgl. DF: 28.

${ }^{2}$ Vgl. auch Börnchen: Kryptenhall, S. 212-215. 
Leipziger Bordell, die ihm mit dem Arm die Wange streichelt, bei eben diesem Namen, den er durch die Bücher des Vaters kennengelernt hatte. ${ }^{3}$ Wie ihr tatsächlicher Vorname lautet, erfährt die Leser*innenschaft nicht.

Anhand von Studien über einen bekannten Weiblichkeitstypus des fin de siècle, des der Femme fatale, von sexuellen Metaphern des Romans sowie von Freuds Schriften wird im vorliegenden Kapitel Zeitbloms Verhalten dem weiblichen Geschlecht, und speziell Esmeralda, gegenüber ausgelotet. Die Darstellung der Erzählinstanz bezüglich dieser Figur stellt dann den Ausgangspunkt für die Analyse der Kompositionen dar, die Hetaera Esmeralda ins Zentrum stellen.

\subsection{Weiblichkeit und Sexualität in Doktor Faustus}

Der Name ,Hetaera Esmeralda ‘ verweist nicht nur auf die gleichnamigen Schmetterlinge im eingangs erwähnten Buch von Adrian Leverkühns Vater, sondern prägt als musikalische Chiffre auch viele Werke des Komponisten und stellt folglich eine Erinnerung an die Begegnung sowie die intime Beziehung mit der Prostituierten aus dem Leipziger Bordell dar. ${ }^{4}$ Darüber hinaus verweist die Esmeralda-Episode sowie die durch den Geschlechtsverkehr mit ihr auf Leverkühn übertragene Syphilis auf die Deutungsperspektive „Nietzsche-Roman“ (Ent: 30), die in der Forschungsliteratur zu Doktor Faustus verschiedentlich erörtert wird. ${ }^{5}$ Ebenfalls weit rezipiert wurde die Erläuterung Thomas Manns, warum in Doktor Faustus der Name des Philosophen expressis verbis vergebens zu suchen ist und was dennoch seiner Biographie entlehnt wurde:

Da ist die Verflechtung der Tragödie Leverkühns mit derjenigen Nietzsches, dessen Name wohlweislich in dem ganzen Buch nicht erscheint, eben weil der euphorische Musiker an seine Stelle gesetzt ist, so daß es ihn nun nicht mehr geben darf; die wörtliche Übernahme von Nietzsches Kölner Bordell-Erlebnis und seiner Krankheitssymptomatik, die Ecce-Homo-Zitate des Teufels, das - kaum einem Leser bemerkliche - Zitat von Diät-Menus nach Briefen Nietzches aus Nizza, oder das ebenfalls unauffällige Zitat von Deussens letztem Besuch mit dem Blumenstrauß bei dem in geistige Nacht Versunkenen. (Ent: 29) ${ }^{6}$

\footnotetext{
${ }^{3}$ Siehe DF: 209.

${ }^{4}$ Am 27. Juni 1944 schreibt Thomas Mann in seinem Tagebuch: „Gearbeitet an XIX (h e a e es)“ (TB2: 27.06.1944, S. 70).

${ }^{5} \mathrm{Vgl}$. Ent: 13 .

${ }^{6} \mathrm{Zu}$ Nietzsches Bordell-Erlebnis vgl. auch GkFA: 19.1, S. 189. Andere Ähnlichkeiten zwischen dem Leben Leverkühns und dem Nietzsches (etwa die Paralyse, die
} 
Des Weiteren schreibt Thomas Mann in den Tagebüchern: „Nietzsche gelesen. Befehlshaberisches über das Geschlechtsleben. (Er hat 2 mal im Leben Verkehr gehabt.)“. 7 Eben dieses letzte Zitat bietet eine gute Überleitung zu den folgenden Darlegungen, jedoch nicht weil sich das vorliegende Kapitel der bereits gut erforschten Perspektive des Nietzsche-Romans widmet. ${ }^{8}$ Vielmehr ist es das im Zitat angesprochene sexuelle Leben Nietzsches, das sich mit Beobachtungen zur Darstellung von Hetaera Esmeralda im Roman gut verknüpfen lässt. Diese konzentrieren sich auf ihre sexualisierte Charakterisierung, die vor allem von Zeitbloms Misogynie, was mehrere Stellen des Romans belegen, diktiert ist. Im ersten Teil soll untersucht werden, inwieweit Esmeralda dem Typus der Femme fatale entspricht. Der zweite Teil befasst sich noch näher mit Zeitbloms misogyner Darstellung, indem u. a. ihre Tonchiffre in die Analyse einbezogen wird. Primär liegt folglich der Fokus dieses Abschnitts und im Allgemeinen dieses Kapitels auf der Darstellung von Weiblichkeit und Sexualität in Doktor Faustus.

\subsubsection{Femmes fatales}

Als Ergänzung zum sechsten Kapitel dieser Arbeit über den „Teufel“ soll durch die Analyse der Charakterisierung von Esmeralda im Roman zugleich diskutiert werden, inwieweit sie neben etwa Kretzschmar und Spengler als Teufelsemissärin gesehen werden kann. Leverkühn lernt diese Figur durch Vermittlung eines weiteren Teufelsemissärs kennen, nämlich durch den Leipziger Dienstmann, mit dem er die Stadt Leipzig besichtigt (es handelt sich wie im Fall des Teufelsgesprächs um ein fiktives Dokument des Komponisten an Zeitblom, diesmal in Form eines auf das Jahr 1905 datierten Briefes). Am Ende des Tages ist Leverkühn erschöpft und hungrig. Der Dienstmann bringt ihn zu einem Gasthaus, das in der Tat ein Bordell ist, und wünscht ihm einen ,Guten Appetit“ (DF: 208). Dem verwirrten und betrogenen Leverkühn kommen einige Prostituierte entgegen, die er folgendermaßen beschreibt:

totale Geisteskrankheit, usw.) wurden u. a. von Bergsten hervorgehoben: Siehe Bergsten: Untersuchungen, S. 72.

${ }^{7}$ TB2: 16.04 .1944$, S. 45

${ }^{8}$ Siehe z. B. Saariluoma, Liisa: Nietzsche als Roman. Über die Sinnkonstituierung in Thomas Manns „Doktor Faustus“. Tübingen: Niemeyer 1996; Klugkist, Thomas: Sehnsuchtskosmogonie. Thomas Manns „Doktor Faustus“ im Umkreis seiner Schopenhauer-, Nietzsche- und Wagner-Rezeption. Würzburg: Königshausen \& Neumann 2000. 
Nymphen und Töchter der Wüste, sechs oder sieben, wie soll ich sagen, Morphos, Glasflügler, Esmeralden, wenig gekleidet, durchsichtig gekleidet, in Tüll, Gaze und Glitzerwerk, das Haar lang offen, kurzlockig das Haar, gepuderte Halbkugeln, Arme mit Spangen, und sehen dich mit erwartungsvollen, vom Lüster gleißenden Augen an. (DF: $208 \mathrm{f}$.)

Leverkühn bezeichnet sie sofort mit dem Namen der Schmetterlinge aus dem Buch seines Vaters, ist verwirrt und sucht Schutz in der Musik, und zwar an einem offenen Klavier:

[Ich] schlage im Stehen zwei, drei Akkorde an, weiß noch, was es war, weil mir das Klangphänomen gerade im Sinne lag. Modulation von H- nach C-dur, aufhellender Halbton-Abstand wie im Gebet des Eremiten im Freischütz-Finale, bei dem Eintritt von Pauke, Trompeten und Oboen auf dem Quartsextakkord von C. (DF: 209)

Gleich danach nähert sich ihm ,eine Bräunliche, in spanischem Jäckchen, mit großem Mund, Stumpfnase und Mandelaugen“ (DF: 209), die ihm mit dem Arm die Wange streichelt und die er Esmeralda nennt (Leverkühns Bezeichnung für alle Prostituierten des Bordells). Noch verwirrter verlässt Leverkühn den Ort so schnell wie möglich. Der Bericht der Begegnung mit Esmeralda, zentraler Ausgangspunkt vieler Beiträge zu Thomas Manns Doktor Faustus, umfasst im Roman nur eine Seite. Die Kommentare des Erzählers umrahmen diesen Bericht: Vor der Wiedergabe von Leverkühns Brief präzisiert etwa Zeitblom, dass er ihn ,,mit Empfindungen las, wie sie wohl eine Mutter bei solchen Mitteilungen eines Kindes bewegen mögen“ (DF: 203). Der Sprachstil ist - wie nicht selten im Fall von Leverkühns fiktiven Dokumenten oder Reden $-{ }^{9}$ reich an Attributen und wirkt exzentrisch, wie die anfängliche Anredeformel „Ehrbar, hochgelahrter, lieber, günstiger Herr Magister und Ballisticus!“ (DF: 204) zeigt. Im letzten Teil des Briefes stellt Leverkühn seine Gedanken bezüglich verschiedener Komponisten, Künstler und Schriftsteller vor, die insgesamt mehr Platz als die Erzählung vom Bordell-Erlebnis einnehmen. ${ }^{10}$

Zusätzlich zu den kurzen Kommentaren vor und nach Leverkühns Brief widmet sich Zeitblom im darauf folgenden ca. sechsseitigen Kapitel noch ausführlicher der Bordell-Episode: Vergleichbar einer Briefausgabe mit Erläuterungen liefert der Erzähler seiner Leser*innenschaft sämtliche Ausführungen zum Inhalt, Sprachstil und zur korrekten Interpretation des Briefes seines Freundes:

\footnotetext{
${ }^{9}$ Dies trifft allerdings oft auch auf Zeitbloms Narrationsstil zu. Vgl. Kap. 7.

${ }^{10} \mathrm{Vgl}$. DF: $210 \mathrm{f}$.
} 
Alles übrige war Zutat, Einhüllung, Vorwand, Aufschub und, nachher, ein gesprächiges Wiederzudecken mit musikkritischen Aperçus, als ob es nichts gewesen wäre. Auf die Anekdote, um ein sehr sachliches Wort zu gebrauchen, steuert alles zu [...]. (DF: 211 f.; Herv. i. O.)

Zeitblom suggeriert, dass ,die Betrachtungen über Schumann, die Romantik, Chopin“ (DF: 212), die den zweiten Teil des Briefes bilden, „offenbar den Zweck“ (ebd.) verfolgen, der sogenannten Anekdote „das Gewicht zu nehmen [...] [und] sie wieder in Vergessenheit zu bringen“ (ebd.). Die Unzuverlässigkeit der Erzählinstanz lässt sich zu Beginn des Kapitels erneut feststellen: „Der kategorischen Weisung, diesen Brief zu vernichten, bin ich nicht gefolgt“ (DF: 211). Nicht nur kommentiert Zeitblom wieder Ereignisse, bei denen er nicht anwesend war, mit großer Sicherheit und Ausführlichkeit, nicht nur gibt er zu, den Brief wie eine besorgte Mutter gelesen und ihn mit zitternden Händen abgeschrieben zu haben, er verrät seiner Leser*innenschaft darüber hinaus, den Willen seines Freundes aufgrund des ,,dokumentarischen Charakter[s]“ (DF: 194), den der Text hat, ignoriert zu haben. Noch komischer wirkt es zudem, dass er seiner Leser*innenschaft eine Analyse des Briefes liefert, deren Grenzen er jedoch zugleich deklariert: „Analyse hat notwendig den Anschein der Kühle, auch wenn sie im Zustande tiefer Erschütterung geübt wird. Erschüttert aber war ich, mehr noch, ich war außer mir“" (DF: 213). ${ }^{11}$ Seine Analyse entspricht seiner eigenen Definition zufolge aufgrund ihres emotionalen Charakters nicht der Kategorie einer objektiven Interpretation.

Zeitbloms Erschütterung lässt sich dadurch begründen, dass Esmeralda seine erste Konkurrentin in Sachen Liebe und Sexualität darstellt. Dass Leverkühn sexuell begehrt, darüber hat der Erzähler den Leser*innen gegenüber noch nichts verlauten lassen: „Es war, um mich emphatisch auszudrücken, wie wenn man einen Engel über die Sünde sich ergehen hörte“" (ebd.). Der Dogmatismus des katholischen Zeitblom setzt einen einmaligen Bordellbesuch mit einer Sünde gleich. Provoziert vom Bericht des Freundes, gesteht er, eine fast rein sexuelle Beziehung „zu einem Mädchen aus dem Volk“ (DF: 215) unterhalten zu haben. Dann äußert er seine tiefe Eifersucht, indem er zugleich Indizien für sein Verhalten Frauen gegenüber liefert:

Ich hatte Lust, die Hexe mit dem Knie von ihm wegzustoßen, wie er den Schemel beiseite stieß, um den Weg ins Freie zu gewinnen. Tagelang spürte ich die Berührung ihres Fleisches auf meiner eigenen Wange und wußte dabei mit Widerwillen, mit Schrekken, daß sie seither auf der seinen brannte. (DF: 217)

\footnotetext{
${ }^{11}$ Noch ein Beweis dafür, dass Zeitblom ein unzuverlässiger, aber doch kein inkompetenter Erzähler ist. Vgl. 7.1.2.
} 
Nach dem ersten Treffen mit Esmeralda im Leipziger Bordell, fährt Leverkühn im Mai 1906 nach Graz, um ,die österreichische Première der ,Salome““ (DF: 224) zu hören. Dies ist zumindest das, was er seinen Freunden und seinem Lehrer Kretzschmar erzählt. Es bleibt jedoch unklar, ob er an der Aufführung überhaupt teilgenommen oder lediglich Esmeralda besucht hat. Die Prostituierte, die krank ist und sich in Pressburg befindet, erinnert sich noch an Leverkühn und warnt ihn vor ihrem Körper; Leverkühn ,verschmäht[]“ (DF: 226) aber die Warnung und besteht ,auf dem Besitz dieses Fleisches“ (ebd.). So soll Leverkühn zum ersten Mal Beischlaf mit einer Frau gehabt und sich folglich mit Syphilis infiziert haben. ${ }^{12}$

Die Wahl der Oper geht Hand in Hand mit Esmeraldas Charakterisierung im Roman. ${ }^{13}$ Denn Salome ist Stefan Wurz zufolge ,geradezu der Inbegriff der Femme fatale“; ${ }^{14}$ Silvia Volckmann fügt hinzu, ihr Phantasma sei für viele Künstler*innen und Schriftsteller*innen zur Verkörperung ,einer blutigen Bedrohung durch das Weib und seine verführerische Potenz"15 ${ }^{* 15}$ geworden. Das Motiv der Versuchung durch die Frau antizipierte schon Privatdozent Schleppfuß in seinen Theologie-Sitzungen. ${ }^{16}$ Darüber hinaus wurde die Figur Salome in der Literatur

\footnotetext{
${ }^{12}$ Die Stadien dieser Krankheit werden im folgenden Beitrag beschrieben: Lahmann, Claas: Die Bedeutung der Krankheit für die Kunst in Thomas Manns Roman „Doktor Faustus“. Frankfurt am Main: Fischer 1995.

${ }^{13}$ Vorausgesetzt selbstverständlich, dass man davon ausgeht, dass sie nicht Zeitbloms Phantasie entspringen. Auch der junge Adolf Hitler soll laut Wimmer an der Premiere teilgenommen haben. Vgl. GkFA 10.2: $426 \mathrm{f}$.

${ }^{14}$ Wurz, Stefan: Kundry, Salome, Lulu. Femmes fatales im Musikdrama (Magisterarbeit Karlsruhe 1999, Karlsruher Beiträge zur Musikwissenschaft Bd. 4). Frankfurt am Main (u. a.): Lang 2000, S. 84. Zur Femme fatale vgl. auch Bronfen, Elisabeth: Liebestod und Femme fatale. Der Austausch sozialer Energien zwischen Oper, Literatur und Film. Frankfurt am Main: Suhrkamp 2004; Hoffmann-Curtius, Kathrin: Constructing the „femme fatale": A Dialogue between Sexology and the Visual Arts in Germany around 1900. In: Fronius, Helen (Hrsg.): Representation of female victims and perpetrators in German culture 1500-2000. Rochester/NY: Camden House 2008, S. 157-185. Zu Femmes fatales bei Thomas Mann siehe: Galvan, Elisabeth: Femme fatale und Allegorie. Thomas Manns Renaissancedrama „Fiorenza“ und das München der Jahrhundertwende. In: Koopmann, Helmut (Hrsg.): Die Wiederkehr der Renaissance im 19. und 20. Jahrhundert. Münster: mentis 2013, S. 181-193.

${ }^{15}$ Volckmann, Silvia: Die Frau mit zwei Köpfen. Der Mythos Salomé. In: Kreuzer, Helmut (Hrsg.): Don Juan und Femme fatale. München: Fink 1994, S. 127-142, hier: S. 128.

${ }^{16}$ Vgl. DF: 155 u. 6.1.1.
} 
auch mit der Syphilis verbunden, da der Teufel durch sie in dem (von Thomas Mann selbst kritisierten) Drama Das Liebeskonzil von Oskar Panizza die Krankheit verbreitet. ${ }^{17}$

Wie lassen sich Femmes fatales definieren? Helmut Kreuzer bezeichnet sie als „Typus der kollektiven Phantasie“18 und hält sie für ,mythische Figuren, für fiktive Figuren, für ideologieträchtige Symbolfiguren der Erotik, die das eine gemeinsam haben, daß sie dem Mann zum Verhängnis zu werden drohen, der in ihren weiblichen Bannkreis gerät" ${ }^{19}$ Auch für Wurz ist die Femme fatale eine

verhängnisvolle Frau [...], die meist unter Einsatz weiblicher Reize einen Mann ins Unglück stürzt, bzw. zu stürzen versucht. Dieser kommt dabei häufig zu Tode, verliert aber mindestens seine Gesundheit, seinen Verstand, seinen gesellschaftlichen Status oder seine Familie. ${ }^{20}$

Beide Definitionen lassen an Doktor Faustus denken. Esmeralda muss wenig weibliche Reize einsetzen, um Leverkühn zu erregen und zu verwirren: ihre laszive Bekleidung, die in ihrer Durchsichtigkeit den Körper zugleich kleidet und zeigt $^{21}$ sowie die Berührung mit dem Arm. ${ }^{22}$ Zeitblom spricht bezüglich der Liebe seines Freundes zu ihr von einer ,kruden Fixierung der Begierde auf ein bestimmtes und individuelles Ziel; ich sehe sie in dem Moment der Wahl, sei diese auch unfreiwillig und von ihrem Gegenstande dreist provoziert" (DF: 224; Herv. i. O.). Die Folgen der Ansteckung sind gesundheitliche Probleme sowie der Wahnsinn, die beide zum definitiven Verlust von Leverkühns gesellschaftlichem Status und zum Tod führen. Nur wenige Bekannte und Freund*innen nehmen an

\footnotetext{
${ }^{17}$ In demselben Drama gibt es außerdem eine andere Femme fatale, und zwar Lucrezia Borgia. Panizza, Oskar: Das Liebeskonzil: Eine Himmelstragödie in 5 Aufzügen, Zürich: Schabelitz 1894. Die Kritik Thomas Manns an dem Stück findet sich in: Mann, Thomas: Das Liebeskonzil [Rez.]. In: Das zwanzigste Jahrhundert 5 (1895) H. 5, S. 522. Des Weiteren wird Esmeralda in der Forschungsliteratur auch mit Wagners Kundry assoziiert, der Dienerin des Grals, die Esmeralda laut Puschmann in dieser Doppelrolle von Verführung und ,selbstloser Hilfsbereitschaft“ gleiche. Puschmann: Magisches Quadrat, S. 144. Siehe auch Börnchen: Kryptenhall, S. 254.

${ }^{18}$ Kreuzer, Helmut: Einleitung. In: Ders. (Hrsg.): Don Juan und Femme fatale, S. 7-16, hier: S. 9.

${ }^{19}$ Ebd.

${ }^{20}$ Wurz: Kundry, Salome, Lulu, S. 17.

${ }^{21}$ Siehe Börnchen: Kryptenhall, S. 249-252.

${ }^{22}$ Vgl. DF: 209.
} 
seiner Beerdigung teil: lediglich Zeitblom, Schildknapp, Jeanette Scheurl, Kunigunde Rosenstiel, Meta Nackedey und eine „unkenntlich verschleierte Fremde“ (DF: 738).

Der Typus - oder mit Nieberle genauer gefasst: das „kulturelle[] Stereotyp[]“23 - ,Femme fatale“ lässt sich nicht eindeutig beschreiben, jedoch seien im Folgenden drei Konstanten erwähnt: Sie ist exotisch, eine Tänzerin bzw. eine Sängerin und steril. ${ }^{24}$ Auch in Doktor Faustus kommt es nicht zwecks der Reproduktion zum Geschlechtsverkehr mit Esmeralda. Der Hinweis in der Abschiedsrede auf das Kind, das Leverkühn mit Hyphialta gehabt habe und das man häufig mit Echo assoziiert, scheint eine Erfindung des wahnsinnigen Komponisten zu sein; es sollte zudem geklärt werden, ob die These plausibel ist, dass Hyphialta und Esmeralda dieselbe Figur sind. ${ }^{25}$ Man weiß selbstverständlich nicht, ob Esmeralda tatsächlich an Sterilität leidet, jedoch lässt sich der Intimverkehr zwischen Leverkühn und ihr auf einer nicht metaphorischen Ebene (denn auf dieser Ebene ist er doch die Quelle erneuter künstlerischer Inspiration) als steril begreifen; auch bezüglich Leverkühns unverheirateter oder verwitweter Verehrerinnen (Frau von Tolna, Nackedey, Rosenstiel, Scheurl) ist festzustellen, dass im Roman nur beiläufig die Rede von ihren Kindern ist. ${ }^{26}$

Darüber hinaus stellt Esmeralda eine exotische Figur dar (das Adjektiv wird hier in seiner ursprünglichen, Fremdländisches bzw. Ausländisches bezeichnenden Bedeutung verwendet). Die Übersetzung des deutschen Wortes ,Smaragd“ ins Spanische lautet ,Esmeralda ${ }^{6}{ }^{27}$ Dies ist aber nicht das einzige Element, das die Prostituierte mit Spanien verbindet. Sie wird von Leverkühn als „eine Bräunliche, in spanischem Jäckchen, mit großem Mund, Stumpfnase und Mandelaugen“ (DF: 209) beschrieben. Des Weiteren begegnet er der Frau zum zweiten Mal in Pressburg, dem heutigen Bratislava, also in einer Stadt, die damals ebenfalls außerhalb Deutschlands lag. Esmeralda singt und tanzt nicht. Dennoch nähert sie sich Leverkühn im Leipziger Freudenhaus, nachdem er völlig verwirrt einige Akkorde am Klavier angeschlagen hat: ${ }^{28}$ Damit entsteht ein Zusammenhang mit der Musik.

\footnotetext{
${ }^{23}$ Nieberle, Sigrid: Gender Studies und Literatur: Eine Einführung. Darmstadt: Wissenschaftliche Buchgesellschaft 2013, S. 58.

${ }^{24}$ Siehe Wurz: Kundry, Salome, Lulu, S. 34.

${ }^{25}$ Vgl. DF: 725. Siehe dazu auch die Frage nach der Identität von Frau von Tolna in 10.1.2.

${ }^{26}$ Scheurl betreffend, siehe etwa DF: 294.

${ }^{27}$ Siehe Oswald, Victor A. Jr.: Thomas Mann's Doktor Faustus: The Enigma of Frau von Tolna. In: Germanic Review 23 (1948) H. 4, S. 249-253, hier: S. 252.

${ }^{28}$ Vgl. DF: ebd.
} 
Die Femme fatale repräsentiert Carola Hilmes zufolge eine „männliche[] Wunsch-Angst-Projektion“. ${ }^{29}$ Das heißt, so erläutert das Ulrich Meier, die Bedrohung komme nicht wirklich von außen, sondern es finde eine „Verteufelung der Frau“30 statt: „Man wirft dem Weib die Urheberschaft ungestümer Regungen, allgemein ein Bündnis mit unterirdischen Kräften vor". ${ }^{31}$ Dies scheint genau in Thomas Manns Roman der Fall zu sein: Zeitblom spricht bezüglich des Geschlechtsverkehrs von „Verlangen nach dämonischer Empfängnis“ (DF: 226) und er betrachtet die Frau u. a. im Teufelsgespräch als Teufelsemissärin. ${ }^{32}$

Der Typus der Femme fatale stellt als Reaktion auf die Emanzipation und das 1918 eingeführte Wahlrecht für die Frau ein typisches Kunstprodukt der Jahrhundertwende dar. ${ }^{33}$ Nicht selten hat sie die Rolle der Prostituierten inne oder wird als solche porträtiert: „Die dämonische Verführerin“, so Hilmes, ,ist Heilige und Hure in einer Person und verkörpert als gefallener Engel einen verteufelten Eros“. ${ }^{34}$ Rudloffs Analyse scheint zu einem ähnlichen Schluss zu kommen: Dort sei Doktor Faustus ein „Zwei-Brüder-Märchen“; 35 in diesem Kontext lasse sich Leipzig, Stadt des Bordellbesuchs, mit einem „Zauberwald“36 vergleichen, ,in dem sich der Fremde verirrt ${ }^{\text {*37 }}$ und in dem Esmeralda die doppelte Funktion von Hexe und Helferin übernimmt. Sie könne für eine Hexe gehalten werden, da sie durch Verzauberung die Krankheit auf einen Helden überträgt, aber auch für eine Helferin, weil der Held danach innovativer komponieren kann. ${ }^{38}$

\footnotetext{
${ }^{29}$ Hilmes, Carola: Kleopatra. Das versteinerte Frauenbild und die Geschichten eines verteufelten Eros. In: Kreuzer (Hrsg.): Don Juan und Femme fatale, S. 99-116, hier: S. 100.

${ }^{30}$ Meier, Ulrich: Verführerinnen der Jahrhundertwende. Kunst - Literatur - Film. In: Kreuzer (Hrsg.): Don Juan und Femme fatale, S. 155-163, hier: S. 156.

${ }^{31}$ Ebd., S. 159.

${ }^{32}$ Vgl. DF: 341. Darmaun findet, Esmeralda sei eine Art Mephistophela. Siehe Darmaun: Thomas Mann, Deutschland und die Juden, S. 269.

${ }^{33}$ Vgl. Meier: Verführerinnen, S. 156.

${ }^{34}$ Hilmes: Kleopatra, S. 105.

${ }^{35}$ Rudloff: Hetaera Esmeralda, S. 404.

${ }^{36}$ Ebd.

${ }^{37}$ Ebd.

${ }^{38}$ Vgl. ebd., S. 404.
} 


\subsubsection{Unvollständige Tonchiffren und Zeitbloms Misogynie}

Es gilt nun, die Verteufelung von Esmeralda und vielen anderen Figuren weiblichen Geschlechts durch die Erzählinstanz näher auszuloten. Laut Börnchen gliedern sich die weiblichen Figuren von Doktor Faustus in sexualisierte und desexualisierte Gestalten. ${ }^{39}$ Es zeichnen sich folglich zwei Darstellungsarten im Roman ab: Entweder erfüllen Frauen im Roman Reproduktionszwecke bzw. sie bedienen einfach das Verlangen nach sexueller Befriedigung des Mannes oder sie bieten ihm Schutz und sind unattraktiv. Das wahrscheinlich beste Beispiel aus dem Roman, das im Rahmen von Börnchens Studie eine wichtige Rolle spielt, ist Zeitbloms Ehefrau, Helene, die nicht nur den Namen des mythischen Inbegriffs der Frau, die aus trojanischer Sicht ins Unglück bringt, trägt, sondern viele weibliche Tugenden verkörpert. ${ }^{40}$ Exemplarisch sei hier ein Zitat aus dem zweiten Kapitel von Doktor Faustus erwähnt, in dem der Erzähler, nachdem er Leverkühn kurz vorgestellt hat, sich selbst präsentiert:

Frühzeitig, bald schon nach meiner Bestallung in Kaisersaschern, habe ich mich vermählt - Ordnungsbedürfnis und der Wunsch nach sittlicher Einfügung ins Menschenleben leiteten mich bei diesem Schritt. Helene, geb. Ölhafen, mein treffliches Weib, das noch heute meine sich neigenden Jahre betreut, war die Tochter eines älteren Fakultäts- und Amtskollegen zu Zwickau im Königreich Sachsen, und auf die Gefahr hin, das Lächeln des Lesers hervorzurufen, will ich nur gestehen, daß der Vorname des frischen Kindes, Helene, dieser teure Laut, bei meiner Wahl nicht die letzte Rolle spielte. Ein solcher Name bedeutete eine Weihe, deren reinem Zauber man nicht seine Wirkung verwehrt, sollte auch das Äußere der Trägerin seine hohen Ansprüche nur in bürgerlich bescheidenem Maß, und auch dies nur vorübergehend, vermöge rasch entweichenden Jugendreizes erfüllen. Auch unsere Tochter, die sich längst einem braven Manne, Prokuristen an der Filiale der Bayerischen Effektenbank in Regensburg, verbunden hat, haben wir Helene genannt. Außer ihr schenkte meine liebe Frau mir noch zwei Söhne, so daß ich die Freuden und Sorgen der Vaterschaft nach Menschengebühr, wenn auch in nüchternen Grenzen erfahren habe. (DF: $20 \mathrm{f}.)^{41}$

„Perfekter“ könnte Zeitbloms Frau nicht sein: Sie ist kein „Mädchen aus dem Volke“ (DF: 215) wie das, mit dem er in Halle eine rein sexuelle Beziehung

\footnotetext{
${ }^{39}$ Siehe Börnchen: Kryptenhall, S. 314.

${ }^{40}$ Vgl. ebd., S. 204 ff.; Ní Dhuíll, Caitríona: Biographie von ,er‘ bis ,sie‘. Möglichkeiten und Grenzen relationaler Biographik. In: Fetz, Bernhard (Hrsg.): Die Biographie - Zur Grundlegung ihrer Theorie. Berlin (u. a.): de Gruyter 2009, S. 199-226, hier: S. 212.

${ }^{41}$ Vgl. auch DF: 251: „Heute morgen, während Helene, meine gute Frau, uns den Morgentrank bereitete [...]“.
} 
unterhalten hatte, sie betreut ihn und hat ihm darüber hinaus auch männliche Erben geschenkt. Kein Wunder, dass sich das Ehepaar für denselben Namen bei der Wahl des Tochternamens entschieden hat, der sich - sittlich-bürgerlichen Verhältnissen gemäß - als erfolgsversprechend erwiesen hat. Des Weiteren wird im obigen Zitat nochmals die mitberücksichtigte klangliche Qualität der von Zeitbloms verwendeten Wörter betont. ${ }^{42}$ Zeitbloms Frau kommt aus Sachsen, was für Börnchens These einer „Helena Esmeralda““, 43 also einer Identität von Helene und Esmeralda, sprechen könnte, da in Leipzig der Bordellbesuch angesiedelt ist.

Was für intellektuelle Fähigkeiten Zeitbloms Ehefrau und Tochter besitzen, erfährt die Leser*innenschaft nicht. Dies ist aber kein Einzelfall im Roman, denn, auch wenn weiblichen Gestalten Intelligenz oder im geistigen Bereich liegende Talente zugesprochen werden, geraten diese wegen der detaillierten, oft zugespitzten Beschreibung von Körpermerkmalen in den Hintergrund; das gilt insbesondere für die sexualisierten weiblichen Figuren von nicht selten niedrigem sozialen Status. Nicht nur wird auf den großen Mund und die Stumpfnase Esmeraldas hingewiesen, ${ }^{44}$ auch die Stallmagd Hanne, die den Kindern das Singen von Kanons beibringt, ist Zeitblom zufolge so ein ,tierisch duftende[s] Geschöpf“ (DF: 39), dass sie an manchen Stellen der Narration sogar den Namen „Stall-Hanne“ (DF: 47) bekommt. ${ }^{45}$ Auch in Pfeiffering soll es eine Stallmagd ,mit Waberbusen und emsig mistigen Barfüßen“ (DF: 46) gegeben haben, die ,der Hanne von Buchel so ähnlich“ (ebd.) sieht. Der Körper weiblicher Figuren steht im Roman stets im Vordergrund, auch der nackte Körper, wie nicht nur an der Beschreibung der Prostituierten im Leipziger Bordell, sondern auch anhand des Namens von Leverkühns Verehrerin Meta Nackedey deutlich wird. ${ }^{46}$

Andererseits gebe es - so z. B. Börnchen - desexualisierte weibliche Figuren in Doktor Faustus, die Leverkühn verehren und ihm auch Schutz bieten. Frau von Tolna ist beispielsweise Leverkühns Mäzenin: Sie finanziert sein Werk, ist bei allen Uraufführungen unsichtbar dabei und ihre Körpermerkmale werden kaum beschrieben. ${ }^{47}$ Sie lässt an einige berühmte weibliche Mäzeninnen der Musikgeschichte denken, z. B. an Frau von Meck, die Mäzenin Tschaikowskis. ${ }^{48}$ Eine

\footnotetext{
${ }^{42}$ Vgl. Kap. 5.

${ }^{43}$ Bornchen: Kryptenhall, S. 262.

${ }^{44}$ Zudem sollte diese Beschreibung von Leverkühn stammen.

${ }^{45}$ Siehe ebd., S. 266.

${ }^{46}$ Vgl. ebd., S. 268.

${ }^{47}$ Auf diese Figur geht 10.1.2. ein.

${ }^{48}$ Vgl. ebd.
} 
weitere desexualisierte Figur wäre Kunigunde Rosenstiel, die Leverkühn in Pfeiffering regelmäßig besucht: Zeitblom spricht von „Huldigungsbesuch[en]“ (DF: 456), Rosenstiel konkurriert mit einer anderen Verehrerin Leverkühns, nämlich mit der bereits erwähnten Meta Nackedey, die schon durch ihren Nacktheit ausdrückenden Namen den sexualisierten weiblichen Figuren zugeordnet werden kann. Börnchens dichotomischer Untergliederung in sexualisierte und desexualisierte weibliche Figuren lässt sich auch anhand der folgenden textuellen Indizien widersprechen: Das Erste, was Zeitblom über Rosenstiel sagt, ist, dass sie einerseits „Mit-Inhaberin eines Darmgeschäftes“ (DF: 455), andererseits ,jüngferlich[]“ (ebd.) wie die Nackedey ist. Der Erzähler stellt auch hier, ähnlich wie bei der Beschreibung der Stallmägde, Aspekte und Körpermerkmale weiblicher Figuren, die zu einem impliziten Vergleich zwischen der Tierwelt und Frauen führen, in den Vordergrund ${ }^{49}$ und spricht sofort die sexuelle Sphäre an. ${ }^{50}$ Zwar präzisiert er gleich danach, was er mit „Darmgeschäft“ (DF: 455) meint („,will sagen: eines Betriebes zur Herstellung von Wursthüllen“, ebd.), zuvor werden jedoch innere Organe angesprochen, die nicht weit entfernt von den Reproduktionsorganen liegen. Durch die explizite Erwähnung, dass sie eine Jungfrau ist, wird die sexuell-konnotierte Rezeptionslenkung aufrechterhalten. ${ }^{51}$ Diese taucht auch in den Äußerungen Rosenstiels auf, von denen Zeitblom kurz danach berichtet, denn das „Ach“, mit dem sie alle Sätze anfängt, ${ }^{52}$ ist ja Klagelaut und verweist auf die Weheklag, kann aber auch mit dem Ächzen beim Geschlechtsverkehr in Verbindung gebracht werden und kommt in den Äußerungen des wohl ,sexualisierten“, androgyne Züge tragenden Schwerdtfegers ebenfalls vor. ${ }^{53}$ Diese Beobachtung verleiht dem durchgehenden Klagen im Text eine neue Konnotation und macht noch einmal deutlich, dass Frauen für Zeitblom zwar andere Tugenden besitzen, Rosenstiel sei etwa sehr musikalisch und könne sich ausgezeichnet in

\footnotetext{
${ }^{49}$ Das gibt Zeitblom selbst zu: ,[D]enn eine Wurstdarmfabrik hat entschieden etwas Derbes“ (DF: 456).

${ }^{50}$ Dazu siehe auch: Stephan, Inge: „Da werden Weiber zu Hyänen ...“: Amazonen und Amazonenmythen bei Schiller und Kleist. In: Stephan, Inge u. Sigrid Weigel (Hrsg.): Feministische Literaturwissenschaft. Dokumentation der Tagung in Hamburg vom Mai 1983. Berlin: Argument 1984, S. 23-42.

${ }^{51}$ Zur Figur vgl. auch Elsaghe, Yahya: Kunigunde Rosenstiel. Thomas Manns späte Allegorie des jüdischen „Volks“. In: Germanisch-Romanische Monatsschrift. Neue Folge 51 (2001) Heft 2, S. 159-172 u. Ders.: Die Jüdinnen in Thomas Manns Erzählwerk. In: Monatshefte 93 (2001) H. 2, S. 169-175.

${ }^{52}$ Siehe DF: 456. Vgl. auch Börnchen: Kryptenhall, S. 313.

${ }^{53}$ Vgl. DF: 429 u. Kap. 9.
} 
der deutschen Sprache ausdrücken, ${ }^{54}$ hauptsächlich aber der Reproduktion bzw. Befriedigung männlichen Begehrens dienen.

Kaum anzuzweifeln ist die Sexualisierung Esmeraldas: Sexuelle Metaphern und erotische Motive prägen bereits das dritte Kapitel von Doktor Faustus, in dem der gleichnamige Schmetterling präsentiert wird. Dort ist nicht nur von diesen Insekten die Rede, sondern auch von Muscheln und Schnecken: Die Formen beider Tiere sind nicht selten mit weiblichen Intimorganen assoziiert worden; zudem wird der „Giftbiss[]“ (DF: 30) einer besonderen Art von Schnecken im Kapitel beschrieben, der sich mit der späteren Übertragung der Syphilis durch den Geschlechtsverkehr in Verbindung bringen lässt. ${ }^{55}$ Außerdem stellt Leverkühn in der kompositorischen Phase der Gesta romanorum seine Absicht eines „Durchbruch[s] [...] aus geistiger Kälte in eine Wagniswelt neuen Gefühls“ (DF: 468; Herv. i. O.) vor und antizipiert somit die spätere revolutionäre Lösung der Zwölftontechnik als Ausweg aus der Sterilität der Kunst, indem er auf die sexuelle Penetration anspielt, was typographisch durch die Kursivierung auch hervorgehoben wird. ${ }^{56}$ Dies führt zu metamedialen Reflexionen, die - darauf weist Börnchen hin - vom ,topologischen Hintergrund des ,Wahrheits“ begriffes “57 ausgehen: Dieser werde z. B. bei Heidegger und Derrida allegorisch mit dem Frauenkörper und mit der Ausübung einer gewissen Gewalt zwecks Entkleidens der Oberfläche des Textes verbunden. ${ }^{58}$ Auch der Topos der Sterilität der Musik zielt auf die Reproduktionsunfähigkeit der Musik und rekurriert auf sexuelle Metaphern: In dieser Hinsicht soll auch die Musik ,durchdrungen“ werden; der Durchbruch, den Leverkühn zur Zeit der Gesta beabsichtigt, ermöglicht ihm laut Zeitblom zum „Geheimnis der Musik“ (DF: 549) in der Apocalipsis cum figuris zu gelangen. Diese so entdeckte „Wahrheit der Musik“ soll dann Leverkühn in der Weheklag umgesetzt haben; kein Wunder, dass sich der Erzähler dort folgende Fragen stellt:

Bedeutet es nicht den „Durchbruch“, von dem zwischen uns, wenn wir das Schicksal der Kunst, Stand und Stunde derselben, besannen und erörterten, so oft als von einem Problem, einer paradoxen Möglichkeit die Rede war, - die Wiedergewinnung, ich möchte nicht sagen und sage es der Genauigkeit willen doch: die Rekonstruktion des Ausdrucks, der höchsten und tiefsten Ansprechung des Gefühls

\footnotetext{
${ }^{54}$ Siehe DF: 457.

${ }^{55}$ Vgl. Börnchen: Kryptenhall, S. 215.

${ }^{56}$ Wörter, die eine sexuelle Konnotation besitzen, erscheinen im Roman oft kursiv, wie an den Romanzitaten des vorliegenden Kapitels festzustellen ist.

${ }^{57}$ Börnchen: Kryptenhall, S. 264.

${ }^{58}$ Siehe ebd. u. Derrida: Grammatologie. Frankfurt am Main: Suhrkamp 2016 [Paris 1967], 13. Aufl., S. 23-35.
} 
auf einer Stufe der Geistigkeit und der Formenstrenge, die erreicht werden mußte, damit dieses Umschlagen kalkulatorischer Kälte in den expressiven Seelenlaut und kreatürlich sich anvertrauende Herzlichkeit Ereignis werden könnte?

Ich kleide in Fragen, was nichts weiter als die Beschreibung eines Tatbestandes, der seine Erklärung im Gegenständlichen sowohl wie im Künstlerisch-Formalen findet. (DF: 703)

Lexikalisch tauchen hier wieder Wörter auf, die auch Leverkühn zum Beschreiben seines künstlerischen Vorhabens verwendet hatte (,Durchbruch', ,geistig“, ,Gefühl', ,Kälte‘); durch das Verb ,müssen“ wird darauf hingewiesen, dass es sich hier um keine optionale, sondern um eine „obligatorische Alternative“59 handelt. ${ }^{60}$ Diese erlaube die Überwindung der Sterilität der Kunst und die Aufdeckung ihres wahren Wesens, das die Sprache nur in Fragen zu kleiden vermag. Daher auch die Wiederholung der Motive der Durchsichtigkeit (etwa die Kleider der Prostituierten in Leipzig) und der Verschleierung (etwa die verschleierte Frau bei Leverkühns Trauerfeier): Börnchen spricht diesbezüglich von einem ,topische[n] Schleier, der durchsichtig gemacht, aufgehoben oder gar zerrissen werden muß, um zur ,Wahrheit" dessen zu gelangen, was sich dahinter befindet “61 dieser Schleier gehöre eindeutig einer Frau. ${ }^{62}$ Die Präsenz dieser allegorischen Motive im Roman, die mit dem Topos der Sterilität der Kunst einhergehen, ${ }^{63}$ stellen die auch von Adorno angesprochenen Vorwürfe eines übertriebenen Intellektualismus der Neuen Musik in Frage. ${ }^{64}$ Die Entwicklung der Dodekaphonie wird metaphorisch mit einem Geschlechtsakt assoziiert, der mit einem gewissen Widerstand vollzogen wird: Zwar soll Esmeralda Leverkühn ,alle Süßigkeiten ihres Weibtums“ (DF: 226) aufgeboten haben, ,um ihn zu entschädigen für das, was er für sie wagte“ (ebd.), aber die Prostituierte wirft sich nicht in die Arme des Komponisten, sondern warnt ihn ,vor ihrem Körper“ (DF: 225; Herv. i. O.). ${ }^{65}$

\footnotetext{
${ }^{59}$ Manzoni: Anmerkungen, S. 56.

${ }^{60}$ So auch Manzoni in seinen Schriften bezüglich Leverkühns Umsetzung der Zwölftontechnik. Vgl. 5.2.1.1.

${ }^{61}$ Börnchen: Kryptenhall, S. 263.

${ }^{62}$ Siehe ebd.

${ }^{63}$ Börnchen weist darauf hin, der Transparenz- und Diaphaniebegriff sei die Trope für die Durchdringung. Siehe ebd., S. 250.

${ }^{64}$ Siehe PhnM: 21 f. Auch Adorno verknüpft die Idee eines Durchbruchs mit der einer (gesellschaftlichen) Wahrheit. Siehe ebd., S. 24.

${ }^{65}$ Daher die These in der Forschungsliteratur, Leverkühn infiziere sich absichtlich. Vgl. 6.1.2 Hier steht wieder die sexuelle Dimension im Zentrum und die Textstelle wird typographisch durch die Kursivierungen erneut hervorgehoben.
} 
Esmeraldas Warnung lässt sich auch mit jenem von Freud in Jenseits des Lustprinzips beschriebenen Reizschutz in Verbindung bringen. Zur Beschäftigung mit diesem Text motiviert einerseits das chronotopische textuelle Indiz des Geschlechtsaktes mit Esmeralda - Leverkühn reist 1906 nach Graz, um dann weiter nach Pressburg zu fahren, der Schauplatz ist die Monarchie Österreich-Ungarn - andererseits die Notwendigkeit, sich mit den damaligen die Sexualität betreffenden psychoanalytischen Theorien auseinanderzusetzen. Dies verbindet sich mit der Absicht zu prüfen, ob auch lediglich auf lexikalischer Ebene ähnliche Begriffe auftauchen, die die sexuelle Metaphorik des Textes noch deutlicher machen. ${ }^{66}$ In Bezug auf den Reizschutz spricht Freud von einer ,,besondere[n] Hülle oder Membran“, 67 die ,,reizabhaltend wirkt [...] bis nicht Reize von solcher Stärke herankommen, daß sie den Reizschutz durchbrechen“. 68 Dieser Reizschutz kommt im Roman sowohl durch die Warnung Esmeraldas als auch durch das Motiv der Verschleierung zum Tragen: Dieser kann Freud zufolge durchbrochen werden, wobei die Folge dieses Durchbruchs ,die gemeine traumatische Neurose“69 sei. Des Weiteren schildert Freud die Sexualtriebe als Lebenstriebe, ${ }^{70}$ die sich gegen die Ich-Triebe, also die Todestriebe, opponieren: ${ }^{71}$ Diese seien zugleich ,lebenserhaltend und verjüngend“72 und könnten ,,narzißtisch“, 73 insbesondere wenn die „Libido vom Objekt abgezogen und aufs Ich gerichtet wird“ ${ }^{74}$ sadistisch, wenn

${ }^{66}$ Die hier betrachtete Schrift von Freud erschien 1920, als die Monarchie ÖsterreichUngarn nicht mehr bestand. Der Erzähler Zeitblom berichtet aber retrospektiv während des Zweiten Weltkriegs und der Roman selbst wurde 1947 veröffentlicht, sodass man von einer Rezeption von Freuds Theorien wohl ausgehen kann. Zum Einfluss von Freuds Schiften auf Thomas Manns Roman siehe: Ceppo, Anna Maria: Freud nel „Doctor Faustus“ di Thomas Mann. In: Nuova Rivista Storica 64 (1980) H. 5, S. 637-652; Straus, Nina Pelikan: „Why Must Everything Seem Like Its Own Parody?“: Thomas Mann's Parody of Sigmund Freud in Doctor Faustus. In: Literature and Psychology 33 (1987) H. 3-4, S. 59-75.

${ }^{67}$ Freud, Sigmund: Jenseits des Lustprinzips. Leipzig (u. a.): Internationaler Psychoanalytischer Verlag 1921 [1920], 2. durchgesehene Aufl., S. 24.

${ }^{68}$ Ebd.

${ }^{69}$ Ebd., S. 28 Auf derselben Seite taucht auch direkt das Substantiv ,Durchbruch“ auf. Es wundert daher in diesem Kontext nicht, dass die Konsequenz jenes Durchbruchs von Leverkühn, der sowohl der Geschlechtsverkehr mit Esmeralda als auch die Entdeckung der Zwölftontechnik sein könnte, eben das Delirium ist.

${ }^{70}$ Siehe z. B. ebd., S. $38 \mathrm{f}$.

${ }^{71}$ Vgl. etwa ebd., S. 43.

${ }^{72}$ Ebd., S. 49.

${ }^{73}$ Ebd.

${ }^{74}$ Ebd., S. 51. 
der Trieb, ,auf die Schädigung des Objektes zielt“" ${ }^{75}$ aber auch masochistisch „als eine Rückwendung des Sadismus gegen das eigene Ich“"76 gewendet werden.

In den Romankapiteln, die auf die Bordell-Episode folgen, häufen sich Wörter und Ausdrücke, die auch in Freuds Text zu finden sind und die auch der sexuell-konnotierten Deutungsebene des Durchbruchs- und Verschleierungsmotivs zugeordnet werden können. So taucht beispielsweise mehrfach das Wort „Trieb“ auf (z. B. DF: 226, auch „,nackte[r] Triebe“", was wieder auf das Motiv der Nacktheit hinweist, DF: 224). Das Wort „Begierde“ (DF: 224) ist ebenfalls zu finden; des Weiteren vergleicht Zeitblom Leverkühns Bericht vom Bordell-Erlebnis mit einer ,Durchbrechung einer sonst unbedingten und von mir stets respektierten Verschlossenheit" (DF: 214; Herv. A. O.). Der Erzähler definiert diesen Trieb als eine „krude Fixierung der Begierde auf ein bestimmtes und individuelles Ziel“ (DF: 224; Herv. i. O.), was also der Auffassung Freuds folgend für einen narzisstischen Trieb sprechen könnte. Sexualität kombiniere sich hier mit dem Bereich des Dämonischen:

\begin{abstract}
Und, gütiger Himmel, war es nicht Liebe auch, oder was war es, welche Versessenheit, welcher Wille zum gottversuchenden Wagnis, welcher Trieb, die Strafe in die Sünde einzubeziehen, endlich: welches tief geheimste Verlangen nach dämonischer Empfängnis, nach einer tödlich entfesselnden chymischen Veränderung seiner Natur wirkte dahin, daß der Gewarnte die Warnung verschmähte und auf dem Besitz dieses Fleisches bestand? (DF: 226)
\end{abstract}

Der Ausdruck „Besitz dieses Fleisches“ weist noch einmal darauf hin, dass es sich um einen narzisstischen Trieb handelt: Ob die Frau dem Geschlechtsakt zustimmt, scheint hier und an anderen Stellen der Esmeralda-Kapitel irrelevant zu sein, nicht zuletzt, weil gar keine Frau erwähnt wird, denn Esmeralda wird hinter dem Wort „Fleisch“ verborgen. Das weibliche Geschlecht erfüllt daher lediglich Reproduktionszwecke und wird in der Narration zum sexuellen Objekt eines begehrenden Mannes gemacht. ${ }^{77}$ Eine gewisse Misogynie Zeitbloms lässt sich auch anhand eines Vergleichs der Tonchiffren von Leverkühn und Esmeralda konstatieren. Dass die Tonchiffre Esmeraldas in mehreren Kompositionen Leverkühns auftaucht oder sogar zum Ausgangsmaterial des Komponierens gemacht

\footnotetext{
${ }^{75}$ Ebd., S. 53.

${ }^{76}$ Ebd., S. 54.

${ }^{77}$ Leicht unterschiedlich profiliert sich aber im Roman die Figur Ines Rodde, der sich u. a. Kap. 9 widmet.
} 
wird, ${ }^{78}$ ist wohl bekannt. ${ }^{79}$ In der Tat ist diese musikalische Chiffre falsch oder genauer gesagt - unvollständig. Hetaera Esmeralda wäre: h e a e a es e a d a. Man könnte aufgrund der Wiederholung der Töne auch schreiben: h e a e (a) es (e) (a) d (a) und dementsprechend vier Töne eliminieren. Die wiederholten Töne dazwischen sollen laut Zeitblom in einigen Kompositionen Leverkühns vorkommen, von dieser vergessenen Note d ist aber nie die Rede: Der Name lautete - übersetzt in Buchstaben - ,Hetaera Es ${ }^{،}{ }^{80}$ Esmeraldas Tonchiffre ist daher defizitär und stellt ihren sozialen Status in den Vordergrund; es mag auch nicht erstaunen, dass die Intervalle, aus denen Leverkühns und Esmeraldas Tonchiffren bestehen, zum großen Teil vergleichbar sind, bis auf jene verminderte Prime, die nur in der Tonchiffre der weiblichen Figur zu finden ist (Abbildung 8.1):

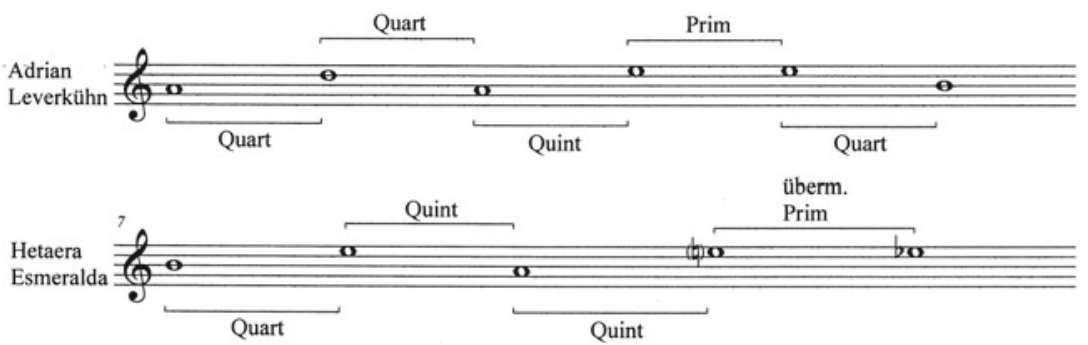

Abbildung 8.1 Die Tonchiffren Leverkühns und Esmeraldas

Die explizite Erwähnung der Tonchiffre Esmeraldas regt zu diesem Vergleich mit nicht explizit benannten Tonchiffren an. Zeitbloms oft misogyne Darstellung von weiblichen Figuren wird durch die beiden vorher genannten Elemente, die Auslassung der Note d sowie die Präsenz einer verminderten Prime (die - wie der Name selbst sagt - ein Intervall vermindert, sprich: kleiner macht), weiter

\footnotetext{
${ }^{78}$ Siehe etwa den Brentano-Zyklus, DF: 279.

${ }^{79}$ Vgl. z. B. Weiher, Frank: Die literarische ,Wiedergabe‘ fiktiver Musik, S. 83.

${ }^{80}$ Siehe auch Manzoni: Su Scene sinfoniche, S. 295.
} 
verstärkt. ${ }^{81}$ Die Misogynie der Erzählinstanz kristallisiert sich bereits aus textimmanenter sowie intra- und intermedialer Perspektive heraus; diese Haltung des Erzählers dem weiblichen Geschlecht gegenüber kommt in ihrer Darstellung als Femme fatale und in der Darstellung der Frau als Objekt, welches sexuelle Wünsche erfüllt, zum Tragen. Im Roman wird ihr zudem eine unvollständige Tonchiffre gegeben.

\subsection{Vom Roman zur Musik}

Nachdem im ersten Teil des Kapitels untersucht wurde, wie Esmeralda im Roman durch die Erzählinstanz Zeitblom dargestellt wird, soll im Folgenden auf die Darstellung dieser Figur in vier Kompositionen eingegangen werden, nämlich im zweiten Stück von Elaines Fines Four pieces from „Doktor Faustus“, in Henzes Violinkonzert, in Hetaera Esmeralda von Claude Lenners und in Manzonis Oper.

\subsubsection{Walzer und $h$ e a e es d: Elaine Fines Esmeralda}

Das zweite Stück von Elaine Fines Four pieces from „Doktor Faustus“ (für Viola d'amore und Klavier) ${ }^{82}$ konzentriert sich, wie der paratextuelle Hinweis des Titels deutlich macht, auf die Figur Esmeraldas. Die Komponistin beschreibt ihr Werk so: ${ }^{83}$

Hetaere [sic] esmeralda is a waltz that has a tone row as its main melody. It is not a 12-tone piece, but it still has obvious connections to the time and place of the book.

\footnotetext{
${ }^{81}$ Dies könnte zu den Versehen des Romans zählen; diese Frage lässt sich wahrscheinlich lediglich anhand des Kriteriums der Intentionalität sowie einer genauen Rekonstruktion der tatsächlichen Interventionen Adornos beantworten, was den Rahmen und die Methode dieser Arbeit sprengen würde. Börnchen hebt des Weiteren hervor, dass sich in den Ausführungen Leverkühns, die auf die Bordell-Episode folgen, Chopin als männliche, positive Gegenfigur profiliert. Siehe DF: 192 ff. u. Börnchen: Kryptenhall, S. 254.

${ }^{82}$ Zwei Stücke aus der Komposition wurden in 7.2.1 analysiert.

${ }^{83}$ E-Mail an die Verfasserin (02.07.2013).
} 
Raum und Zeit des Romans sollen also laut der Komponistin Ausgangspunkt des Vertonens gewesen sein. Ob sich Fine in „Hetaere esmeralda“ auf das BordellErlebnis in Leipzig oder auf den Geschlechtsakt in Pressburg bezieht, bleibt unklar. Die Musikform des Stückes, die der paratextuelle Hinweis ,Waltz Tempo“ zu Beginn der Komposition offen deklariert, scheint mehr mit der Monarchie Österreich-Ungarn als mit Leipzig zu tun zu haben. Folglich könnte man zur Auffassung tendieren, hier werde Esmeraldas Geschlechtsverkehr mit Leverkühn geschildert. Von Leverkühn ist aber, zumindest in den paratextuellen Hinweisen der Komposition, gar nicht die Rede: ${ }^{84}$ Fines Stück stellt Esmeralda ins Zentrum. Die Viola d'amore scheint hier nicht mehr für Zeitblom, sondern für sie zu stehen, nicht zuletzt, weil ihre Stimme eben mit der Tonchiffre $h$ e a e es beginnt. Alles könnte jedoch auch immerhin von Zeitblom, dem Viola d'amoreSpieler des Romans, erzählt werden. Die Vagheit von Elementen, die Narrativität induzieren, in der instrumentalen Musik lässt zugleich Ambiguitäten und Interpretationspotenziale erscheinen. Jedenfalls hat die Komponistin nicht übersehen, dass die Tonchiffre Esmeraldas im Roman unvollständig ist: „Hetaere Esmeralda“ ergänzt hier den Roman, indem Esmeralda die Note d im letzten Teil ihrer Tonfolge zurückgegeben wird.

Da Pressburg, wie bereits erläutert, 1906 ebenso zur Monarchie ÖsterreichUngarn gehörte wie Graz, die Stadt, in der Leverkühn auf dem Weg nach Pressburg Salome gehört haben soll, liegt nahe, dass die Komposition eine sehr passende Tanzform zur musikalischen Darstellung dieser Figur gewählt hat. Damit unterstreicht sie auch eine weitere Eigenschaft der Femme fatale, die in Doktor Faustus durch Esmeraldas Annäherung an Leverkühn beim Anschlagen einiger Akkorde am Klavier lediglich angedeutet wird: Das Tanzen als typische Ausdrucksform dieses Frauentypus. Der Walzer hat aber keinen Unterhaltungscharakter, sondern erinnert eher an eine valse triste und an die Schwermütigkeit der gleichnamigen Schmetterlinge in Jonathan Leverkühns Bücher (Abbildung 8.2):

Die musikalischen Eigenschaften von „Hetaere esmeralda“ rufen die Musik verschiedener Komponisten aus Leverkühns Zeit ins Gedächtnis: Tschaikowski mit einigen spätromantischen Stellen, Schönberg, Zemlinsky und die ersten Dissonanzen sowie die ,exotischen“ Klänge Debussys, die gut zur „exotischen“ Esmeralda passen. Die Tatsache, dass Fine hier keine dodekaphonische Musik komponiert hat, muss keinen Kontrast zum Roman bilden, denn in dieser Phase

\footnotetext{
${ }^{84}$ Das betrifft allerdings die gesamte Komposition Fines, die Leverkühn, zumindest in den Paratexten, nie erwähnt.
} 

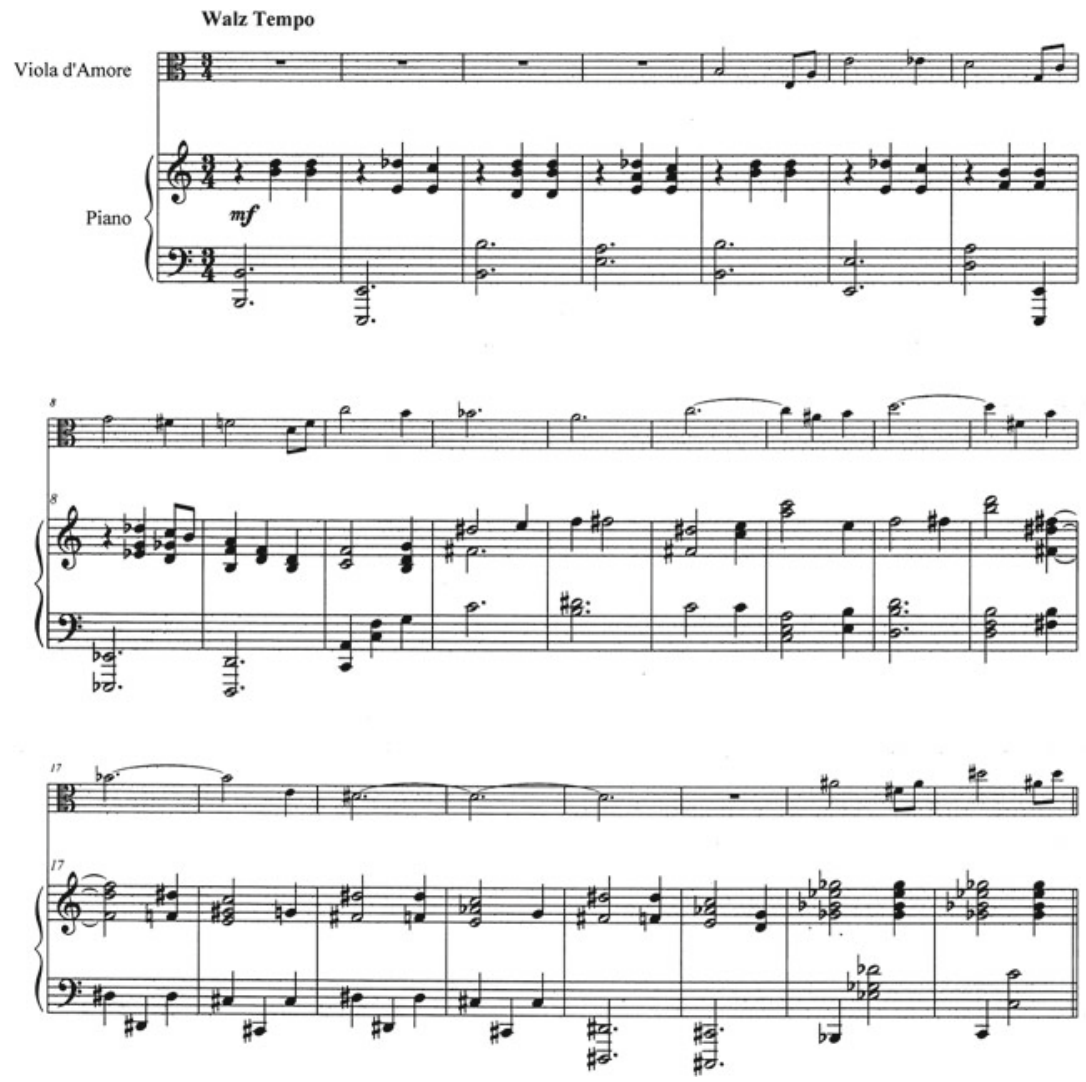

Abbildung 8.2 Der Anfang des Stückes (T. 1-21)

komponiert Leverkühn noch nicht dodekaphonisch. Die instrumentale Komposition ist hier trotz ihres - im Vergleich zu anderen Erzählmedien und -formaten vagen Erzählens in der Lage, Esmeralda als exotisch und musikaffin zu charakterisieren und Raum und Zeit der intradiegetischen histoire wiederzugeben. Diese Aspekte verlieren vielleicht im Medium der instrumentalen Musik an visuellen, gewinnen aber bestimmt an klanglichen Qualitäten, nicht zuletzt weil die Tonchiffre vollständig erscheint. 


\subsubsection{Tango, Flageoletts und Figurencharakterisierung: Hans Werner Henzes 3. Violinkonzert: Drei Porträts aus dem Roman „Dr. Faustus" von Thomas Mann - 1. Satz: Esmeralda}

Das dritte Violinkonzert Hans Werner Henzes (1926-2012) ist dem Geiger Michael Erxleben und dem Berliner Sinfonie-Orchester gewidmet und wurde am 12. September 1997 unter der Leitung von Michael Schønwandt in Berlin uraufgeführt. Es existieren zwei Fassungen des Konzerts: Der ersten von 1997 folgte im Jahr 2002 eine Revision, die aber keine wesentlichen Veränderungen zur ersten Version aufweist. ${ }^{85}$ Die Orchesterbesetzung besteht aus zwei Flöten (die zweite auch Piccolo- und Altflöte), zwei Oboen (die zweite auch Englischhorn), zwei Klarinetten in B (die zweite auch Bassklarinette in B), zwei Fagotten (das zweite auch Kontrafagott), vier Hörnern in F, zwei Trompeten in C, einer Tenorposaune, einer Tuba, Pauken, Schlagzeug (Fingerzimbeln, zwei hängende Becken, zwei Tamtams, Bronzeplatte, große und kleine Trommel, Holzblock, Kastagnetten, Peitsche, Vibraphon und Marimbaphon), Harfe, Celesta, Klavier und Streichern. Es handelt sich in diesem Fall wie auch bei Ruzicka oder bei Fine um verdeckte Intermedialität, da die Komposition auf einem einzigen Medium basiert: Die explizite Systemerwähnung im Titel verweist allerdings unmittelbar auf Thomas Manns Roman, wodurch Henzes Komposition als intermediale Transposition $\mathrm{zu}$ werten ist.

Die Musikwissenschaftlerin Elisabeth Schmierer ordnet das Konzert aufgrund der Wahl eines literarischen Themas dem Genre der sinfonischen Dichtung zu. ${ }^{86}$ Das Interesse des Komponisten an der Literatur spielte in seiner gesamten Produktion eine zentrale Rolle: Henze vertonte nicht nur zahlreiche Werke von diversen Autor*innen, sondern arbeitete auch mit ihnen zusammen, u. a. mit Ingeborg Bachmann. ${ }^{87}$

\footnotetext{
${ }^{85}$ Vgl. Sorg: Beziehungszauber, S. 255. Die revidierte Fassung, auf die sich die Analyse bezieht, erschien 2002 bei Schott, Mainz, die erste 1997 bei demselben Verlag. Die drei Violinkonzerte Henzes findet man in der folgenden Aufnahme: Henze, Hans Werner: Violin Concertos Nr. 1-3, Torsten Janicke (Geige), Magdeburger Philarmonie, MDG 2005. ${ }^{86}$ Siehe Schmierer, Elisabeth: Musik als Sprache. Zu Henzes Instrumentalkonzerten auf literarische Themen. In: Abels, Norbert u. Elisabeth Schmierer (Hrsg.): Hans Werner Henze und seine Zeit. Regensburg: Laaber 2013, S. 279-304, hier: S. 295.

${ }^{87}$ Zur Biographie bzw. Autobiographie Henzes siehe auch: Rosteck, Jens: Hans Werner Henze. Rosen und Revolutionen. Berlin: Propyläen 2009; Henze: Reiselieder mit böhmischen Quinten. Autobiographische Mitteilungen 1926-1995. Frankfurt am Main: Fischer 1996. Vgl. auch Bielefeldt, Christian: Hans Werner Henze und Ingeborg Bachmann:
} 
Bevor der Esmeralda gewidmete Satz aus dem Violinkonzert analysiert wird, gilt es - insbesondere im Rahmen einer intermedial angelegten Studie - auf Henzes Auffassungen zum Verhältnis von Musik und Sprache einzugehen. Diese stellen nämlich eine weitere Positionierung in der Intermedialitätsforschung dar, selbstverständlich mit der reservatio, dass es sich hier um keine wissenschaftliche Positionierung, sondern um die eines Komponisten handelt. So Henze bezüglich des Verhältnisses von Musik und Sprache in seinen autobiographischen Schriften: ${ }^{88}$

Wir sind darin geübt, Musik als Sprache zu verstehen, wir möchten nun etwas mehr über ihre Eigentümlichkeiten wissen als zuvor, das Mythische in ihr verstehen, das Geheimnis durchleuchten, wollen verhindern, daß das Sprachliche der Musik nicht noch weiter ins Leere gestoßen wird, von den Menschen weg: um dieses Sprachliche zu vertiefen, es zugänglich machen [sic] und nützlich und es als eine notwendige Erweiterung des menschlichen Bewußtseins und seines Ausdrucksvermögens zu begreifen.

\section{Jens Brockmeier erläutert dieses Konzept folgendermaßen: ${ }^{89}$}

Musik wie Sprache zu verstehen. Gleichsam auf Augenhöhe. Und das heißt, Musik bemisst sich nach den gleichen Standards der Verständlichkeit, Empathie und poetischen Präzision - und das schließt Mehrdeutigkeit, Vagheit und Offenheit mit ein $[\ldots]$.

Henze scheint im obigen Zitat, wie Brockmeier ebenfalls unterstreicht, auf Adornos Auffassung in Fragment über Musik und Sprache (1956/57) zu reagieren, denn Adorno sagt diesbezüglich: ${ }^{90}$

Musik ist sprachähnlich. Ausdrücke wie musikalisches Idiom, musikalischer Tonfall, sind keine Metaphern. Aber Musik ist nicht Sprache. Ihre Sprachähnlichkeit weist den Weg ins Innere, doch auch ins Vage. Wer Musik wörtlich als Sprache nimmt, den führt sie irre.

die gemeinsamen Werke. Beobachtungen zur Intermedialität von Musik und Dichtung. Bielefeld: transcript 2003.

${ }^{88}$ Henze: Reiselieder, S. 403 f.

${ }^{89}$ Brockmeier, Jens: Eine Sprache in harter Währung. Die Idee musikalischer Sprachlichkeit bei Hans Werner Henze. In: Hans Werner Henze. Musik und Sprache. Musik-Konzepte 132 (2006), S. 5-25, hier: S. 19, Herv. i. O. Siehe auch Bielefeldt: Hans Werner Henze und Ingeborg Bachmann, S. 37-43.

${ }^{90}$ Adorno: Fragment über Musik und Sprache, S. 71. 
Bereits 1956/57 weist Adorno auf die medialen Differenzen zwischen dem Medium der Musik und dem der Sprache hin, indem er zugleich Überschneidungsfelder, beispielsweise Fachbegriffe wie Tonfall oder Idiom, anführt. Mit der Bezugnahme auf die Vagheit der Musik, speziell der instrumentalen Musik, erscheint Adorno somit als Vorreiter der Intermedialitätsforschung. Henze schlägt vor, die Aufmerksamkeit auf produktive Interdependenzen zwischen Musik und Sprache zu lenken, anstatt sich mit Differenzen zu befassen. Dies setzt voraus, dass man Adornos „Weg ins Innere“ der Musik verfolgt, d. h., dass man sich stärker auf die innere Struktur der Musik konzentriert. Henze scheint im genannten Zitat nicht wirklich dafür zu plädieren, dass Musik wie Sprache verstanden werden könne. ${ }^{91}$ Vielmehr befürwortet er den Blick auf Grenzüberschreitungen und erklärt den Blick auf Grenzziehungen für zweitrangig, weil dieser weniger produktiv sei. ${ }^{92}$ Es mag in diesem Kontext nicht verwundern, dass die große Ausdrucksfähigkeit von Henzes Musik in der Forschungsliteratur immer wieder thematisiert wird. Ausgangspunkt des Komponierens ist Brockmeier zufolge immerhin das musikalische Zeichen, wobei die anderen Zeichensysteme eine integrierende Rolle spielen und das Ziel zu verfolgen scheinen, ,die Imagination des Hörers szenisch-performativ zu organisieren“. ${ }^{93}$ Dies stellt Bielefeldt in Frage, der die Intermedialität als Ausgangspunkt nimmt, was sich mit jener von Wolf als primär bezeichneten Intermedialität in Verbindung bringen lässt, die somit Teil des Werkkonzeptes wäre. ${ }^{94}$ Für die vorliegende Untersuchung ist dies besonders interessant, weil es bedeuten würde, dass sich Henzes Violinkonzert zugleich aufgrund der Konzeption als primäre und zusätzlich, da es sich um eine Transposition von Doktor Faustus handelt, auch als sekundäre Intermedialität bezeichnen lässt. Gleichwohl könnte man auch argumentieren, dass die Analyse von Einzelfällen bestimmte intermediale Kategorien ins Schwanken bringt, was anhand dieser (oder vielleicht aller) Kompositionen Henzes deutlich wird.

Den ersten Kontakt mit Thomas Manns Werk hatte Henze bereits im nationalsozialistischen Deutschland. Zusammen mit einem Freund soll er in Bielefeld heimlich die verbotenen Bücher einer Bibliothek, darunter die der Brüder Mann gelesen haben. ${ }^{95}$ Später wählt der Komponist u. a. aufgrund seiner deklarierten Homosexualität und seiner politischen Haltung, Marino, eine Stadt in der

\footnotetext{
${ }^{91}$ Andere Äußerungen und Kommentare des Komponisten scheinen aber dieser Auffassung zu widersprechen. Siehe Bielefeldt: Hans Werner Henze und Ingeborg Bachmann, S. 42. ${ }^{92}$ Vgl. Kap. 1.

${ }^{93}$ Brockmeier: Eine Sprache in harter Währung, S. 24.

${ }^{94} \mathrm{Vgl}$. 1.1.5.

${ }^{95}$ Vgl. Henze: Reiselieder, S. 37.
} 
Nähe von Rom, als Wohnort. Als Komponist stellt sich Henze gegen die Experimente Nonos und Stockhausens: Seine Musik ist - so Bielefeldt - von einer ,(Quasi)tonalität“96 geprägt. $^{97}$

Der erste Satz des Violinkonzerts, der ca. sieben Minuten dauert, ist der Figur Esmeralda gewidmet. Die Tanzkonnotation dieses Satzes stellt ein zentrales Merkmal dar: Die „Sphäre der Unterhaltungsmusik“"98 ist durch die zweimal wiederholte Angabe „nicht eilen, tänzerisch gemütvoll“ (VK: 1, T. 2 und 19, T. 89), die Bezeichnung des zweiten Teiles als „Wiener Lied“ (VK: 11, T. 42) und des dritten als „Tango“ (VK: 13, T. 49) gegeben. Durch das Wiener Lied - die einzige Form, die nicht auf den Tanz zurückgreift - wird bei Henze, ähnlich wie bei Fine, eine Verbindung mit der Monarchie Österreich-Ungarn hergestellt. Der Tango ist laut Schmierer „reine Augenmusik“: 99 Der Rhythmus erscheine zwar in Harfe und Tuba, werde aber kaum wahrgenommen. Durch diese Tanzform kommt Sorg zufolge das Exotische Esmeraldas als „Prostituierte südländischen Typs“100 zum Ausdruck.

Wie in Fines Komposition wird Esmeralda zur tanzenden Femme fatale; die Unterhaltungsmusik eignet sich außerdem in beiden Kompositionen für die Schilderung des Bordells. Sorg sieht in diesem Stück den Charakter eines Totentanzes, was Klangqualität und Partiturangaben jedoch nicht bestätigen: ${ }^{101}$ Es herrschen Dynamikanweisungen wie „soave“, „dolce“ (VK: 9), „dolcissimo“ (VK: 11) und „,con grazia“ (VK: 14), also „lieblich“, „,üßß“, „,sehr süß“ und ,,anmutig“, die bei einer danse macabre ungewöhnlich wären. Die vorherrschende Atmosphäre ist dank der Harfe und der Celesta sowie der „Flageolett-Quintklänge, die aus einer anderen Welt zu kommen scheinen", ${ }^{102}$ nicht so dramatisch wie bei Fine; die isolierten dramatisch wirkenden Momente könnten im Prozess des Erzählens im Medium der instrumentalen Musik auf die Infizierung anspielen.

Der Satz fängt mit einem Rezitativ der Violine solo an, das keine stabile Dynamik aufweist und Stellen im Forte oder sogar im Fortissimo mit solchen

\footnotetext{
${ }^{96}$ Bielefeldt: Hans Werner Henze und Ingeborg Bachmann, S. 40. Brockmeier spricht diesbezüglich von einer „Spannung von Tonalität und Atonalität“. Brockmeier: Eine Sprache in harter Währung, S. 11.

${ }^{97}$ Timo Sorg unterstreicht biographische und poetologische Parallelen zwischen Thomas Mann und H. W. Henze (insbesondere in Bezug auf das Traditionsverständnis und das Parodieverfahren). Vgl. ebd., S. 260-266.

${ }^{98}$ Schmierer: Musik als Sprache, S. 296.

${ }^{99} \mathrm{Ebd}$.

${ }^{100}$ Sorg: Beziehungszauber, S. 272.

${ }^{101}$ Siehe ebd.

${ }^{102}$ Schmierer: Musik als Sprache, S. 295.
} 
im Piano und mit Flageolett-Tönen alterniert. Hier finden sich in kondensierter, akustischer Form zwei Motive der Esmeralda-Kapitel wieder. Das erste Motiv ist das Begehren und die mit ihm einhergehenden Sexualtriebe, die Leverkühn dazu zwingen, nach der Prostituierten zu suchen: Ihre Dringlichkeit kommt auch hier zum Tragen, denn die Notenwerte werden immer kürzer, die Triolen werden zu Sextolen und Septolen und die anfängliche Metronomangabe macht deutlich, dass das nicht zu langsam gespielt werden soll. An vereinzelten Stellen, die mit Piano-Dynamiken und manchmal Flageolett-Tönen korrespondieren, wird das Tempo langsamer (siehe „meno mosso“ und „nicht eilen, tänzerisch gemütvoll“"). Ein zweites Motiv ist die Durchsichtigkeit, welche auch eine Eigenschaft des gleichnamigen Schmetterlings und der Kleider der Prostituierten im Leipziger-Bordell darstellt, das in Henzes Instrumentation durch Flageolett-Töne realisiert wird (Abbildung 8.3)“. Im Geigenspiel können diese Töne nur durch eine leichte Berührung der Saiten erzeugt werden und sind von einem - so Marianne Rônez - „flötenartigen, etwas gläsernen Klang“103 charakterisiert:

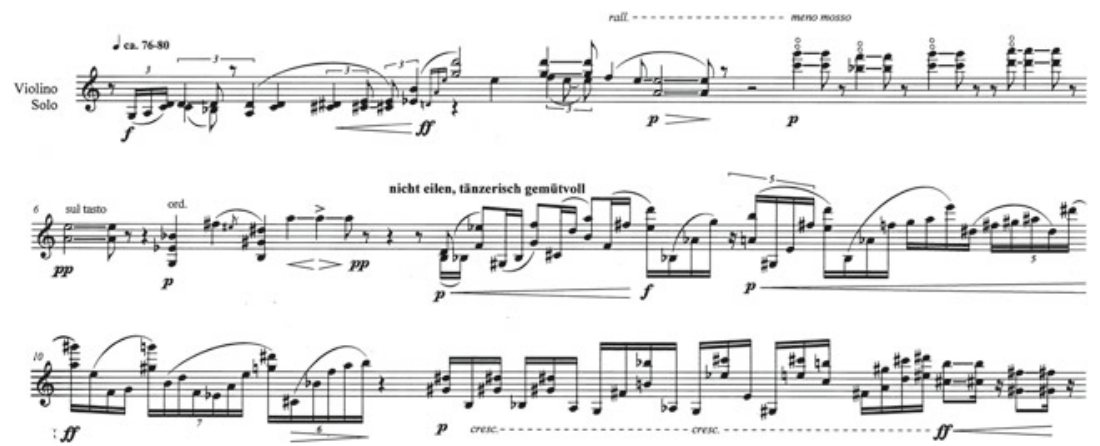

Abbildung 8.3 Der Anfang des Violinkonzerts (VK: 1)

Darüber hinaus können Flageolett-Töne als eine Tonchiffre des Geigenspiels aufgefasst werden, denn sie bedürfen einer Dekodierung: Sehr selten haben sich in der Musikgeschichte Komponist*innen für die als unpraktisch empfundene „Notation von Flageoletts nach dem tatsächlichen Klang“"104 entschieden. Das

${ }^{103}$ Rônez, Marianne: Art. Violine, Violinspiel, Technik, Technik der linken Hand, Flageolett. In: MGG Online. Veröffentlicht 14.09.2015. <https://www.mgg-online.com/mgg/sta ble/49510> (letzter Zugriff: 21.08.2020).

${ }^{104}$ Ebd. 
bedeutet, der graphisch angegebene Ton ist nicht genau der Ton, den man in der Aufführungssituation hört: Auch wenn beispielsweise tatsächlich ein a klingt, korrespondiert dieses nicht mit der graphisch angegebenen Oktave. Flageoletts bedürfen daher einer notationsbedingten Kodierung und einer aufführungsbedingten Dekodierung: Dies kann mit der Kodierung und Dekodierung einer Tonchiffre verglichen werden, die sich aus einem Namen ableitet. Gerade in der ersten Hälfte des 20. Jahrhunderts entstehen viele Violinkonzerte (etwa von Prokof'ev und Schönberg), die auf diese Klänge zurückgreifen und die heute aus zeitgenössischen Kompositionen kaum wegzudenken sind. ${ }^{105}$

Nach dem Einsatz des Orchesters erklingen in Takt 9 Kastagnetten, die deutlich zu hören sind und die die spanischen Eigenschaften der Prostituierten betonen. Im Takt 26 (VK: 8) ist der Einsatz des Klaviers eine deutliche Reproduktion des handlungsbezogenen Moments von Leverkühns Anschlagen einiger Akkorde im Freudenhaus - es ist aber nur der Akt, den die Musik reproduziert, und nicht die im Roman erwähnten Akkorde (Abbildung 8.4): ${ }^{106}$

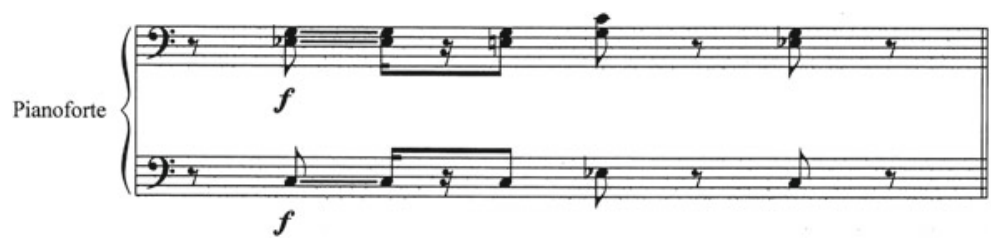

Abbildung 8.4 Die Klavierakkorde im Freudenhaus

Einige Töne der Tonchiffre Esmeraldas, vor allem e und es, sind hier aber zu finden.

Das folgende musikalische Motiv, das sich im Tango-Teil befindet und „flautando" zu spielen ist, verweist wieder, auch graphisch, auf jene Leichtigkeit eines Schmetterlings und seiner Art zu fliegen (Abbildung 8.5):

Die Seiten 17 bis 19 der Partitur sind als Höhepunkt des Satzes aufzufassen. Etwas geschieht hier, wahrscheinlich die intime Beziehung mit der Prostituierten, oder lediglich die Berührung, die auch im Roman den Höhepunkt der Esmeralda-Episode darstellt. Die Dynamik ist im Crescendo $(f, f f$, und $f f f)$; die Tamtams tragen zur bedrohlich-wirkenden Atmosphäre bei. Die Spannung

\footnotetext{
${ }^{105}$ Paradebeispiel hierfür sind die Werke von Salvatore Sciarrino, siehe etwa die 6 Capricci (1975/76) für Violine solo.

${ }^{106} \mathrm{Vgl}$. auch Sorg: Beziehungszauber, S. 270.
} 


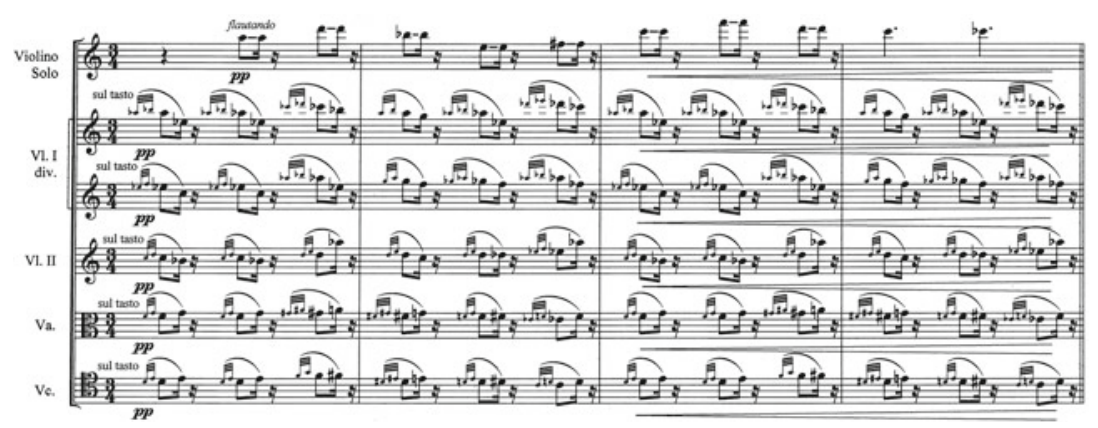

Abbildung 8.5 Der Schmetterling Esmeralda (VK: 14 f., T. 58-61)

wird bis Takt 89 (VK: 19) aufrecht gehalten. Dann spielt die Violine solo eine Kadenz, welche die musikalischen Hauptmotive des Satzes zusammenfasst. Die hier wiederholte Anweisung ,nicht eilen, tänzerisch gemütvoll“" (VK: 19, T. 89) belegt die Kreisstruktur des Stückes. Ab T. 90 (VK: 20) ist die Dynamik im Decrescendo, morendo ( $p p$, ppp und pppp). Die letzten Takte setzen sich aus Flageoletts der Violine solo, einer loopartigen, chromatischen Struktur der Streicher und rhythmischen Figurationen der Kastagnetten zusammen: Sie bilden eine Zusammenfassung von Eigenschaften und Motiven, die man mit Esmeralda, dem gleichnamigen Schmetterling oder im Allgemeinen den Esmeralda-Kapiteln verbindet (die Durchsichtigkeit, die Berührung, die Infizierung, das Exotische, das Fliegen im Kreis eines Schmetterlings).

Schmierer behauptet, Henze verkürze die Tonfolge Hetaera Esmeraldas (h e es), während Ziolkowski zufolge sie nicht benutzt wird. Dieser Auffassung schließt sich auch die vorliegende Studie an, weil die Chiffre in der Komposition kaum identifiziert werden kann und keineswegs programmatisch verwendet wird. ${ }^{107}$ Sucht man im Roman nach einem Vorbild für den musikalischen Stil dieses Satzes von Henze, so ist es vielleicht in ,der symphonischen Phantasie ,Meerleuchten“" (DF: 221) mit jener ,,ausgesuchter Tonmalerei“ (DF: 221) und den ,unenträtselbare[n] Klangmischungen“ (ebd.) zu suchen, die auch wohl diesen Satz aus dem Violinkonzert beschreiben könnten. Berücksichtigt werden muss, dass ein Unterschied zwischen einer Transposition, die sich an den fiktiven Werken Leverkühns orientiert, und einer Transposition, die bestimmte Figuren und Textstellen des Romans in der Musik zu reproduzieren versucht, besteht,

${ }^{107}$ Vgl. Schmierer: Musik als Sprache, S. 296; Ziolkowski: Leverkühn's Compositions, S. 850 . 
sodass es sich in den meisten Fällen nicht lohnt, nach Vorlagen des Komponierens im Roman zu suchen. Dass dies nicht das Hauptvorhaben des Violinkonzerts ist, deklariert bereits der Titel selbst: Dort ist die Rede von Porträts. Der Fokus, der spezifischer als der von Fine mit dem Wort pieces ist, liegt daher in der Figurencharakterisierung und eher in der Beschreibung als im Erzählen bzw. - genauer gefasst - in der Induktion von Narrativität. Die Komposition ruft einzelne Töne aus ihrer Chiffre, ihre durchsichtige Kleidung und den Verweis auf Spanien (im Roman vor allem durch das spanische Jäckchen und ihren spanischen Namen gegeben) klanglich und graphisch in Erinnerung, verweist auf den gleichnamigen Schmetterling und verstärkt wie bei Fine durch den tänzerischen Charakter der Satzteile ihre Assoziation zur musikalischen Welt. Man könnte sagen, dass Zeitbloms Darstellung der weiblichen Figur als Femme fatale hier durch die Wahl verschiedener Tanzformen deutlich unterstützt wird. Die kulturelle Geographie der Monarchie Österreich-Ungarns, auf die lediglich ein Teil in Form eines Wiener Lieds anspielt, ist hier weniger signifikant. Des Weiteren bietet sich musikstilistisch ein Vergleich dieses Violinkonzerts mit denen von Pizzetti und Wolf-Ferrari aus den 1930er/1940er Jahren an. ${ }^{108}$

Der Tango verleiht Esmeralda eine nicht ausschließlich eurozentrische exotische Konnotation, was erneut die Auffassung bestätigt, dass das sekundäre intermediale Produkt aus unterschiedlichen geographischen und historischen Prämissen entsteht, die daher andere Mittel etwa zur Unterstreichung des Exotischen erforderlich machen. Dieser Satz (das gilt allerdings für das ganze Konzert von Henze) bietet ein Beispiel dafür, wie sich Figurencharakteristika im Medium der instrumentalen Musik realisieren lassen. Ob sich Musik, speziell instrumentale Musik, wie Sprache verstehen lässt, kristallisiert sich aus der vorigen Analyse als eine sehr problematische Auffassung heraus, wie auch mit Verweis auf Adornos Zitat deutlich wird. Dieses Bewusstsein für mediale Differenzen, das als Voraussetzung für die Analyse dient, stellt aber keine Einschränkung dar, sondern führt sowohl bei Henze als auch bei Fine zur gezielten Identifikation von Möglichkeiten zur Grenzüberschreitung. ${ }^{109}$

\footnotetext{
${ }^{108}$ Henze hörte Wolf-Ferraris Musik schon in den Bielefelder Jahren. Siehe Henze: Reiselieder, S. 36. Vgl. auch Hamann, Peter: Ermanno Wolf-Ferrari. Tutzing: Schneider 1983. Viagrande, Riccardo: Ildebrando Pizzetti. Compositore, poeta, critico. Monza: Eco 2013. ${ }^{109}$ Siehe Rajewsky: Von Erzählern, die (nichts) vermitteln, S. 39.
} 


\subsubsection{Sichtbare Musik und Zahlensymbolik: Hetaera Esmeralda von Claude Lenners}

Nachdem zwei rein instrumentale Werke vorgestellt wurden, die Esmeralda u. a. durch Flageolett-Töne und Tanzformen charakterisieren, soll im Folgenden auf ein Werk eingegangen werden, das sich zur Darstellung Esmeraldas und der Esmeralda-Episode verschiedener Medien bedient. Hetaera Esmeralda von Claude Lenners (*1956) ist eine Auftragskomposition des Goethe Instituts in Luxemburg, wo sie 1998 aufgeführt wurde. Sie gehört zum Projekt „WORT \& KLANG“, das sich mit den „Dreiecksverhältnisse[n] zwischen Literatur, Musik und bildender Kunst" ${ }^{* 110}$ auseinandersetzt, und ist dem ehemaligen Direktor des Goethe Instituts in Luxemburg, Dr. Paul Eubel, gewidmet. Während der Aufführung wurden einige Stellen aus Doktor Faustus vorgelesen: die Experimente Jonathan Leverkühns, der Bordell-Besuch, die Episode der intimen Beziehung mit Esmeralda, das Teufelsgespräch, die Echo-Episode und die Abschiedsrede. Der Lektüre einer Textpassage folgte ein Musikabschnitt, der sich auf den Inhalt des Gelesenen bezog. Beim Vorlesen wurden einige zentrale Wörter von einem oder mehreren Instrumenten des Ensembles mittels rhythmischer Figurationen oder Klänge betont. ${ }^{111}$ Darüber hinaus sind die verschiedenen Abschnitte, aus denen die Komposition besteht, nicht als separate Elemente aufzufassen, sondern als Teile des Ganzen. Dies ist an der Taktnummerierung sowie am Wiederauftreten einiger musikalischer Motive zu erkennen, die der Komposition Einheitlichkeit verleihen.

Auch waren die Installationen der Künstlerin Margret Lafontaine Teil dieses Projektes, das sich zum Ziel gesetzt hatte, ,den geistigen und kognitiven Kosmos des dichterischen Werkes ins Sichtbare und Hörbare zu verwandeln, doch nicht um Illustrationen geht es hierbei, sondern um Metamorphose: Die Wirklichkeit des Werkes entsteht im Kunstwerk und in der Musik neu“, ${ }^{112}$ erläutert Eubel. Der Roman wurde also in diesem Fall nicht nur musikalisch, sondern auch künstlerisch neu interpretiert: Es handelt sich, da mehrere Medien vorhanden sind, um offene Intermedialität und infolgedessen um eine Medienkombination. Ziel des Werkes ist Eubel zufolge, Doktor Faustus sichtbar und hörbar zu machen. Da sich

\footnotetext{
${ }^{110}$ Eubel, Paul (Hrsg.): Thomas Mann, Claude Lenners, Margret Lafontaine: Hetaera Esmeralda. Luxemburg: Goethe Institut 1998 (Serie Wort \& Klang: Begegnungen von Literatur, Kunst und Musik).

${ }^{111}$ Die Partitur wurde nicht veröffentlicht. Mit freundlicher Genehmigung des Komponisten war es möglich eine Kopie des Manuskripts einzusehen.

${ }^{112}$ Eubel: Thomas Mann, Claude Lenners, Margret Lafontaine, S. 21.
} 
die vorliegende Arbeit in der musikliterarischen Intermedialität verortet, wird das Hauptaugenmerk auf der Musik liegen.

Hetaera Esmeralda wird vom Komponisten als „Destillat des bedeutenden und vielschichtigen Romans ,Dr. Faustus“ von Thomas Mann “113 beschrieben: ${ }^{114}$ Der Komponist weist mit dem Wort „Destillat“ darauf hin, dass es sich um ein partielles intermediales Produkt handelt, das das Resultat einer Auswahl ist. Große Aufmerksamkeit schenkt Lenners der Zahlenmagie: ${ }^{15}$

In diesem Zusammenhang ist auch die Besetzung mit Sopran, 2 Harfen, Glasharfe und Streichtrio zu verstehen, die absichtlich die ungerade 7 in den Vordergrund stellt. Hierbei spielen die beiden Harfen eine zentrale Rolle, weil durch ihre symetrische [sic] Gegenüberstellung die Vision des Glasflüglers zur Geltung kommt.

Auch in diesem Fall ist also die vorherrschende Atmosphäre magisch konnotiert, was nicht nur durch die Zahlensymbolik betont wird, sondern auch durch die Anwendung eines höchst seltenen Instruments wie der Glasharfe. „Insofern soll die Musik den Zuhörer größtenteils in eine geheimnisvolle Stimmung versetzen, die dem Zeitstillstand nahekommt“" ${ }^{116}$ so der Komponist. Zeitstillstand und Geheimnis sollten also die zwei Leitgedanken des Komponierens sein. Die Zahlensymbolik, die auch im Roman eine bedeutende Rolle spielt, etwa kraft des Wiederauftretens der Zahl zwei (bei Lenners mittels der zwei Harfen ebenfalls vorhanden), übernimmt auch hier eine wichtige Funktion, insbesondere in der Gestaltung der Besetzung. Die Sopranstimme steht für Esmeralda: Ihr ist der Schluss des Stückes vorbehalten. Eine Konzentration auf diese Figur lässt sich bereits dem paratextuellen Hinweis des Titels entnehmen, was durch die Besetzung, die um die Sopranstimme kreist, bekräftigt wird. Am Schluss rezitiert die Sopranstimme den in Doktor Faustus erwähnten Text von Clemens Brentano $O$ lieb Mädel, wie schlecht bist du!: ${ }^{17}$ Esmeralda soll - so Lenners - „dabei ihr

\footnotetext{
${ }^{113}$ Zit. in ebd., S. 10.

${ }^{114}$ Zur Biographie von Claude Lenners sei hier auf Weber, Loll: Lenners, Claude. In: Grove Music Online. Zuerst veröffentlicht 20.01.2001, online veröffentlicht 2001. <https:// doi.org/10.1093/gmo/9781561592630.article.45620> (letzter Zugriff: 21.08.2020); Claude Lenners. <https://www.claudelenners.lu/> (letzter Zugriff: 21.08.2020) verwiesen.

${ }^{115}$ Zit. in Eubel: Thomas Mann, S. 10. Die Besetzung enthält außerdem ein reiches perkussives Instrumentarium (Gong, Claves, Waterphone, Vibraphon, Glas Chimes, Rahmentrommel, usw.). Die Glas Chimes sind ebenfalls ein „durchsichtiges“ Instrument. ${ }^{116} \mathrm{Ebd}$.

${ }^{117}$ Vgl. DF: 266. Dieser Text wird auch von Leverkühn vertont.
} 
wahres Gesicht“"118 zeigen. Der Inhalt des Textes verweist wieder auf ,,schlechte“ Frauen, die den Mann ins Unglück treiben; das weibliche Geschlecht verbirgt sich jedoch nicht hinter Tonchiffren oder (misogynen) Darstellungen des Erzählers, sondern kommt direkt zu Wort. Die Sopranstimme Esmeraldas ist auch im Echo gewidmeten Abschnitt zu hören. Auffällig ist, dass die Komponist*innen, welche die Figur der Prostituierten ins Zentrum ihres Stückes rücken, oft auch die des Kindes behandeln: Bei Henze wird ihm der zweite Satz gewidmet, bei Fine das vierte Stück. Gemeinsamer Nenner beider Figuren ist laut Zeitblom, dass sie von Leverkühn geliebt werden und dass diese Liebe sowohl Auswirkungen auf Leverkühns Schaffen als auch seine physische sowie psychische Gesundheit haben.

Wie bei Manzoni herrscht auch bei Lenners eine große Varietät in den Ausdrucksweisen der Stimme (Abbildung 8.6): ${ }^{119}$

Wie in der ersten Beschreibung von Esmeralda im Roman steht auch hier der Mund der Sängerin im Zentrum; die Reichweite der Ausdrucksmöglichkeiten erstreckt sich vom lautlosen Sprechen bis hin zum sehr hohen Schreien: Während Esmeralda im Roman nie direkt zu Wort kommt, wird sie hier zur Hauptfigur, die von ihren Emotionen erzählt. Das Motiv der Verschleierung kommt auch hier zum Tragen, denn die Sängerin soll einen Schleier tragen: Auch wenn sie „mit unverdecktem Gesicht“ spricht, soll dieses Sprechen so lautlos sein, dass „man [...] auf den Lippen ablesen können [soll] was gesagt wird, ohne es zu hören“ (S. III s. obige Abbildung). Dies lenkt die Aufmerksamkeit wieder auf den Mund der Sängerin und kann zugleich als eine Art gesangliche Verschleierung aufgefasst werden, also als eine Realisierung des Motivs mit den Mitteln des Gesanges: Das Gesagte wird vergleichbar dem Topos der nackten Wahrheit gleichzeitig ent- und bekleidet, also mitgeteilt und verdeckt gehalten. Auditiv bleibt die Mitteilung verdeckt, da man sie nicht hören soll, sie wird optisch durch jenes Ablesen auf den Lippen mitgeteilt. Die metaphorische Verknüpfung von Mund und Wahrheit ist auch in der römischen Kunst zu finden (siehe etwa den sogenannten ,Mund der Wahrheit", Bocca della verità, in Rom, was allerdings kein weibliches Gesicht ist). Darüber hinaus findet sich die Idee einer verschleierten Wahrheit auch im mythischen Topos der verschleierten Isis, in diesem Fall eher in Bezug auf die Wahrheit der Natur als auf die des Textes. ${ }^{120}$

\footnotetext{
${ }^{118}$ Zit. in Eubel: Thomas Mann, Claude Lenners, Margret Lafontaine, S. 10.

${ }^{119}$ So der Musikjournalist Loll Weber über die Werke Lenners': „His works display a notable sensitivity to musical colour, and are mostly written for chamber ensembles or orchestra“. Weber: Lenners, ebd.

${ }^{120}$ Dies passt auch sehr gut zum dritten Kapitel von Doktor Faustus, in dem Tiere (darunter auch der Schmetterling Hetaera Esmeralda) und Naturphänomene im Vordergrund stehen,
} 


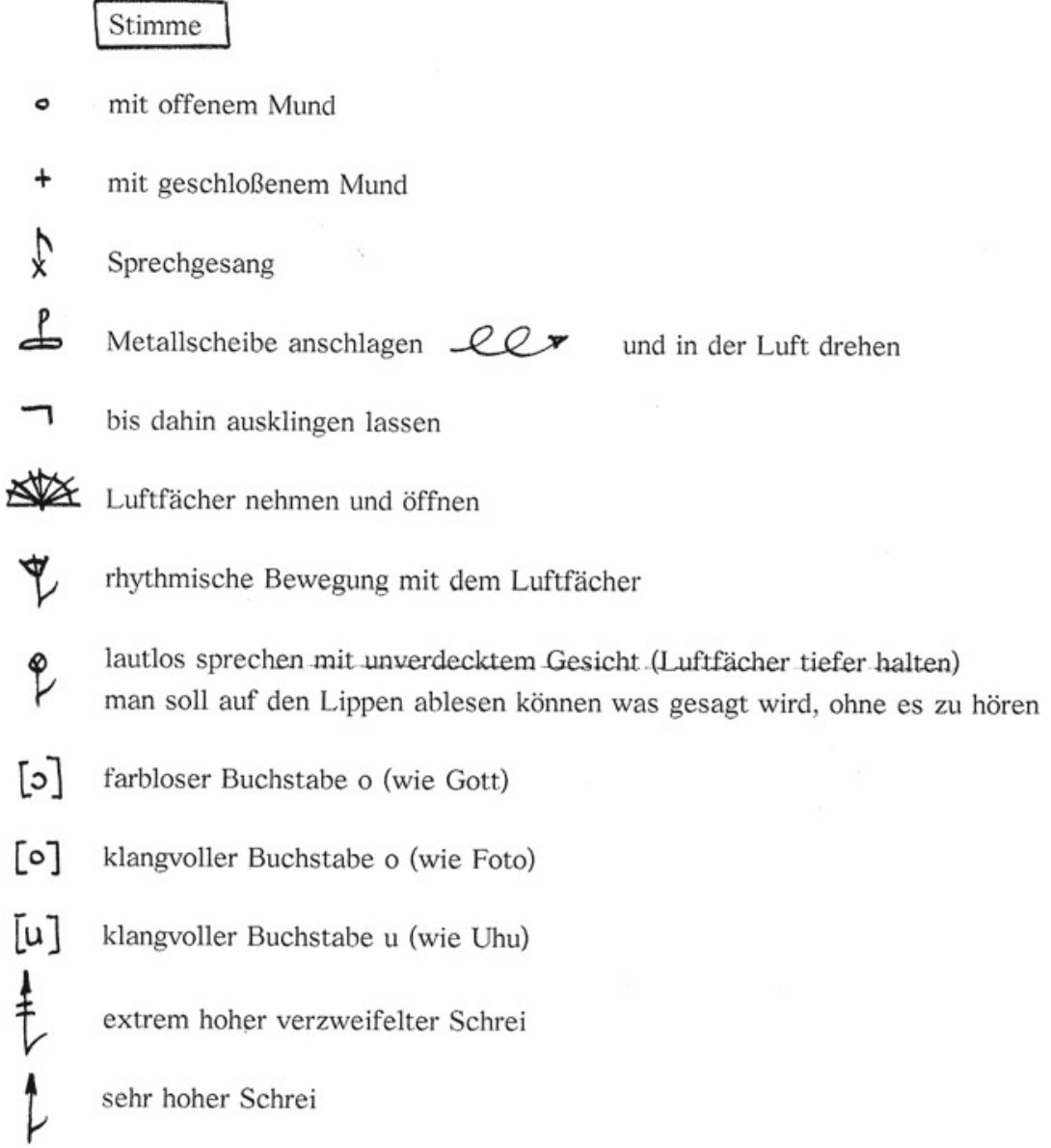

Abbildung 8.6 Anweisungen für die Sängerin (S. III). Mit freundlicher Genehmigung des Autors

und zu dieser Komposition, die nach einer Imitation von Naturklängen strebt. Dazu siehe Assmann, Jan: Immanuel Kant und Friedrich Schiller über Isis und das Erhabene. In: Anselm, Sigrun (Hrsg.): Talismane. Klaus Heinrich zum 70. Geburtstag. Basel (u. a.): Stroemfeld 1998, S. 102-113. 
Die Aufführung beginnt mit der Lektüre einiger Textpassagen aus dem dritten Kapitel des Romans, die vom Inhalt der Bücher Jonathan Leverkühns handeln. Die von den Instrumenten betonten Worte sind: „,öse Geister“, „Malaria“, „Trug“, „Hetaera Esmeralda“ und ,auf ihren Flügeln“. ${ }^{121}$ Die letzten beiden Worte werden durch den Klang der Harfe, die mit dem Schmetterling assoziiert wird, unterstrichen. Der Fokus liegt also auf der trügerischen Schönheit der Natur. Der Lektüre folgt ein Musikabschnitt für zwei Harfen (eine ist links vom restlichen Ensemble, die andere rechts positioniert) und die Glasharfe. Dieser Abschnitt ist „,ben articolato“, „gut artikuliert“ und „misterioso“ (T.1), „geheimnisvoll“ zu spielen: Die vorherrschende Atmosphäre, die dadurch erzeugt wird, ist, genau wie das „misterioso“ aussagt, geheimnisvoll. Der Fokus liegt dabei auf jedem einzelnen gespielten Ton und nicht auf ganzen musikalischen Phrasen. Nicht zufällig ist dieser Abschnitt von einer minimalistischen Musik geprägt: Es gibt nur wenige Elemente, die sich wiederholen. Der Abschnitt basiert auf Esmeraldas Tonfolge, die in der zweiten Hälfte umgekehrt präsentiert wird (ab T. 14). Die Musik ist erneut sowohl akustische als auch optische Musik, was die Positionierung der Harfen, die klanglich und optisch einen Schmetterling reproduzieren sollen, zeigt.

Das nächste Thema sind die Experimente Jonathan Leverkühns: Das der ,,sichtbaren Musik“ (DF: 32) und das des „Fressende[n] Tropfen[s]“ (DF: 33). Das erste Experiment scheint in der Komposition von Lenners nicht nur durch die Lektüre explizit thematisiert, sondern auch durch musikalische Mittel (teil-)reproduziert zu werden, denn ein Leitgedanke von Hetaera Esmeralda ist zweifelsohne der Versuch, Musik auch sichtbar zu machen. Der darauf folgende Musikabschnitt ist reich an Glissandi, die auf den ersten Musikversuch anspielen. Ab T. 46 werden „,die Crotales von den Streichern gespielt, welche sich an 3 verschiedenen Punkten im Raum befinden": Sowohl optisch als auch musikalisch wirkt es so, als wollten die Spieler*innen die Akustik des Raumes testen. Ab T. 62 imitiert das Waterphone die Wassertropfen, die ihr eigenes Tempo haben. Lenners' Komposition zielt darauf ab, Naturphänomene im Medium der Musik zu reproduzieren (Abbildung 8.7):

Der dritte Abschnitt der Lektüre handelt vom Bordell-Erlebnis. ${ }^{122}$ Hier werden die Wörter nicht von den Instrumenten betont. Die Musik am Beginn dieses Abschnitts besteht aus großen Kontrasten: Die Dynamik reicht von einem Forte „so laut wie möglich“123 bis zu einem Pianissimo, das den Zeitstillstand (siehe die Angabe „statico“) erreicht (Abbildung 8.8):

${ }^{121} \mathrm{Vgl}$. DF: 26.

${ }^{122}$ Vgl. DF: 201-211.

${ }^{123}$ Vgl. die allgemeinen Dynamikanweisungen auf S. II des Manuskripts. 


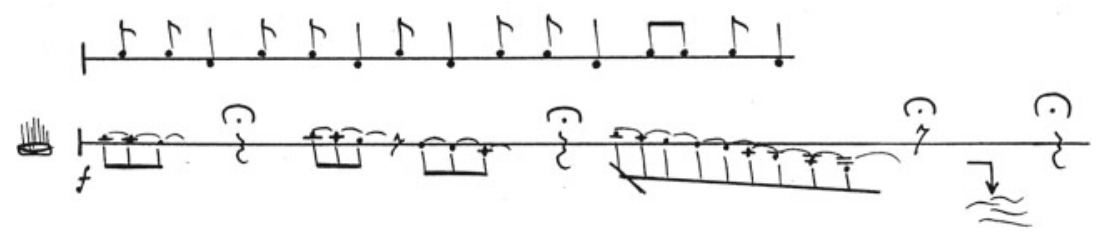

Abbildung 8.7 Die Wassertropfen. Mit freundlicher Genehmigung des Autors

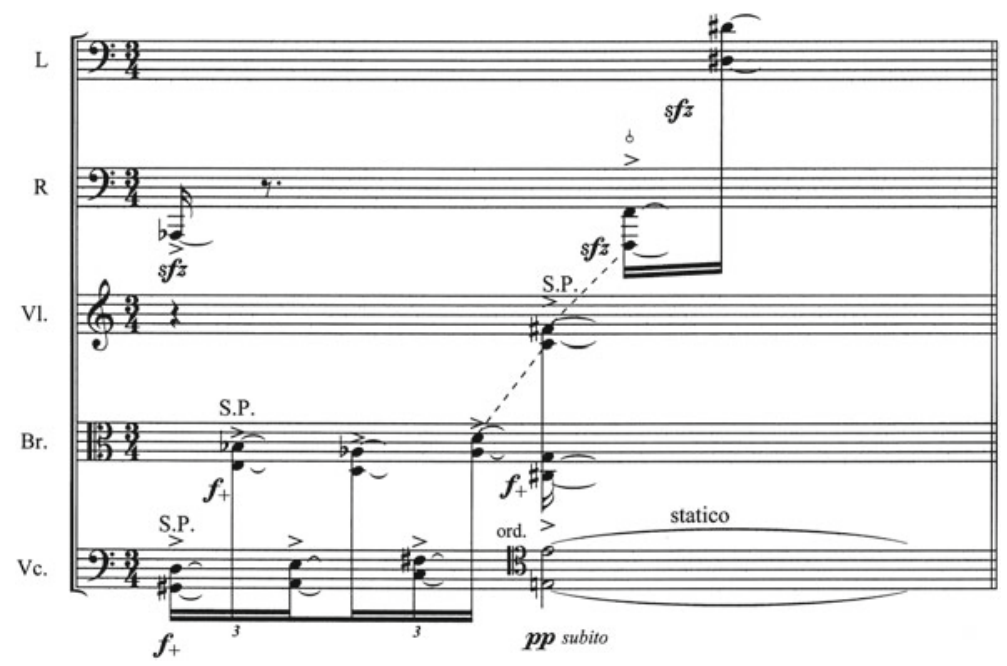

Abbildung 8.8 Der Anfang der Bordell-Episode (T. 74)

Einen ähnlichen musikalischen Verlauf der Glasharfe wie im ersten Abschnitt findet man -leicht variiert - auch hier: Dies stellt das verbindende Element von Hetaera Esmeralda dar. Ab T. 89 beginnt die Sopranistin, ,,beängstigend verführerisch“ „Komm, komm“ zu singen. Auch hier wird das Motiv der Verführung durch die Frau aufgenommen, verstärkt durch die Wiederholung „Komm, komm“: Bei Lenners ermutigt Esmeralda Leverkühn auch durch das wiederholte, gesungene Wort und nicht nur durch die Berührung mit dem Arm, sich ihr anzunähern. Der Höhepunkt ist ab T. 101 zu finden: Der Rhythmus beschleunigt sich, die Dynamik ist reich an Fortissimi und Sforzati und die Noten der Streicher enthalten zahlreiche Glissandi. Die Sängerin soll „,seufzend“ singen. Hier wird entweder 
die Berührung Leverkühns durch Esmeralda oder die Verwirrung des Komponisten nach der Berührung musikalisch illustriert, was die Angabe ,überstürzt“ zu bestätigen scheint (Abbildung 8.9). Zu bemerken ist auch, dass sich ein Glissando bei Saiteninstrumenten eben durch eine gleitende Berührung der Saite bzw. der Saiten erzeugen lässt:

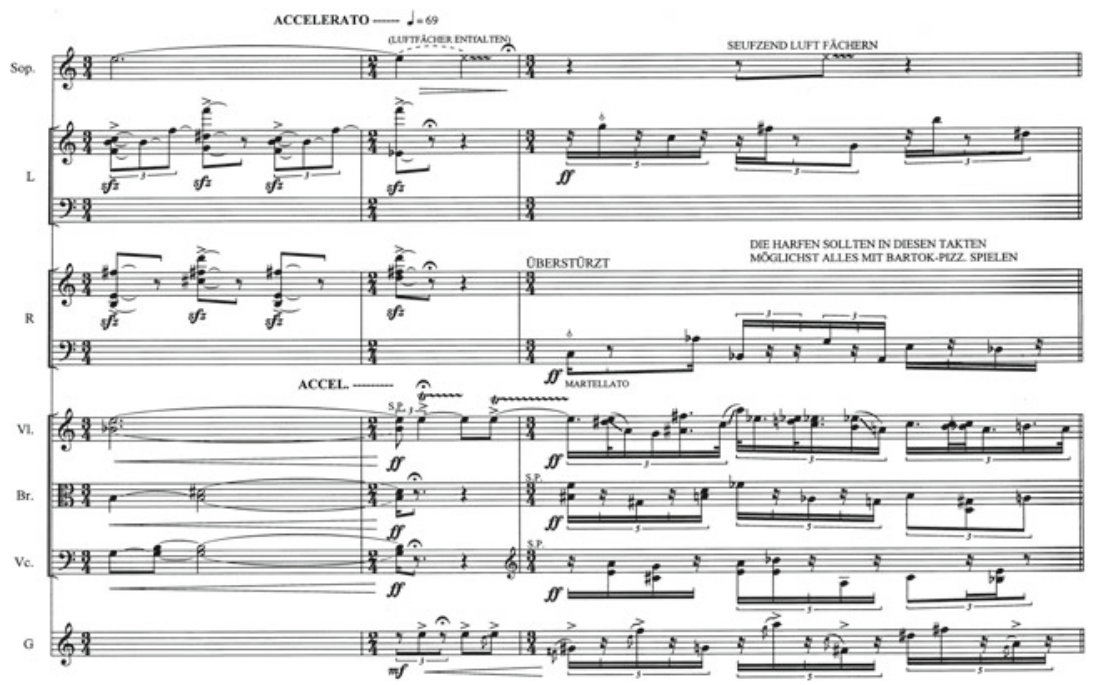

Abbildung 8.9 Die Begegnung mit Esmeralda (T. 101 ff.)

Die Bratsche und das Violoncello schließen diesen Abschnitt mit einem Triller und einem Glissando.

Die vierte Lektüre handelt von der intimen Beziehung in Pressburg. Beim Vorlesen der Wörter „Person“ und „Weib“ ist die Sopranstimme aus der Ferne zu hören. Die Wörter „Streicheln“ und „Trieb“ werden vom Schlagzeug betont und ihre Bedeutung musikalisch (teil-)reproduziert: ${ }^{124}$ Das Motiv des sexuellen Begehrens ist aus der Darstellung dieser Kapitel nicht wegzudenken. Die darauf bezogene Musik schafft Destabilisierung, wie man anhand des ständigen Wechsels der Metronomangaben und Fermaten sowie des unabhängigen Rhythmus der links positionierten Harfe feststellen kann. Ab T. 117 lautet die Dynamikanweisung „,con melencolia“, „melancholisch“. Dies bedarf auch einer Inszenierung: „Die

${ }^{124}$ Vgl. DF: 224 ff. 
Sängerin erscheint jetzt zwischen den zwei symetrisch [sic] aufgestellten Harfen: Esmeralda, der Glasflügler“ (T. 127). Lenners’ Partitur sieht eine Inszenierung vor, zu der nicht nur die Sängerin, sondern auch die Musiker*innen des Ensembles, etwa durch die Glissandi, welche die Berührung zu inszenieren versuchen, beitragen.

Die Warnung Esmeraldas ab T. 145 besteht anfänglich nur aus Vokalen und wird erst im T. 156 zu einem verzweifelten „Geh“ (Abbildung 8.10):
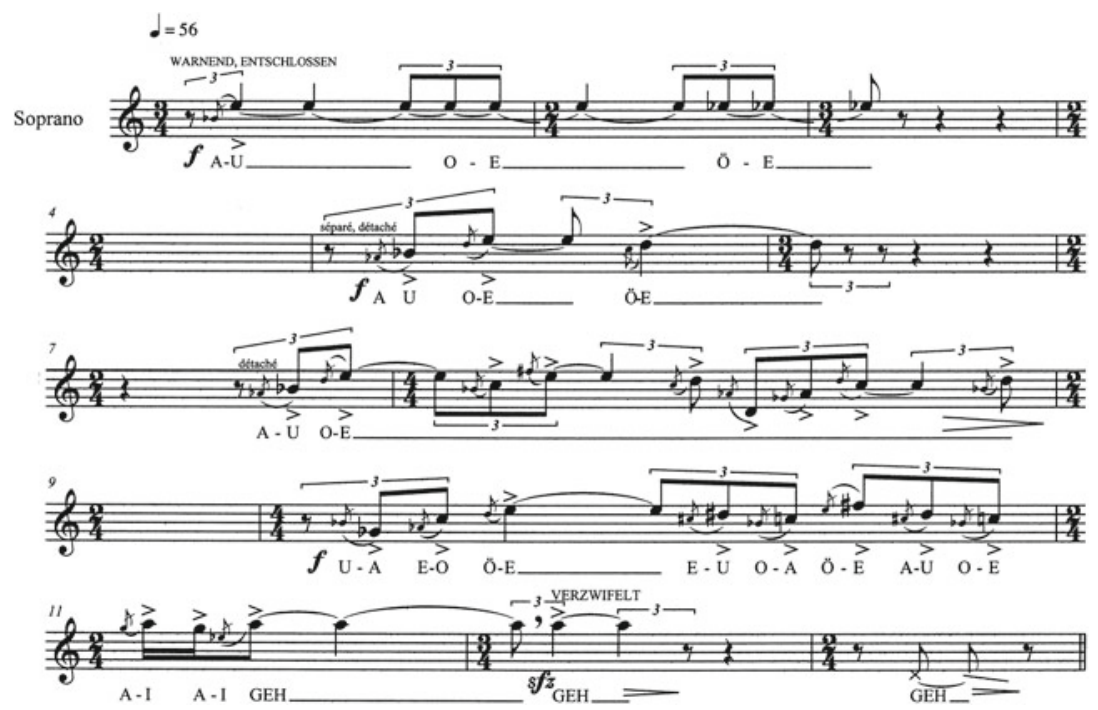

Abbildung 8.10 Die Warnung Esmeraldas (T. 156)

Das imperative „Geh“ ist mehr als Warnung denn als Befehl, der die Aufforderung wegzugehen impliziert, aufzufassen und setzt sich daher dem „Komm, komm“ der Bordell-Episode entgegen. Anfänglich weist also Esmeralda Leverkühn zurück.

Danach wird die Passage vorgelesen, in der es um Zeitbloms Auffassung von „Liebe und Gift“ (DF: 225) geht. Auf Zeitbloms telling und dementsprechend auf seine Wertungen verzichtet Hetaera Esmeralda nicht komplett: Zwar macht sie Esmeralda zur Hauptfigur, jedoch lässt sie durch die Lektüre Zeitbloms Wiedergabe zu Wort kommen und schafft somit einen kommentierenden/diegetischen 
Rahmen im Werk. Der darauf folgende Musikabschnitt beginnt mit der Rahmentrommel und einen Takt danach folgt der Sprechgesang der Sopranstimme. In T. 180 wiederholt die Sängerin: „Komm, komm“. Diesmal soll sie „lautlos sprechen mit unverdecktem Gesicht“ (S. 3), was in der Partitur präzisiert wird: „Man soll auf den Lippen ablesen können, was gesagt wird, ohne es zu hören“ (ebd.). Hier weist Esmeralda Leverkühn nun nicht mehr zurück und so besteht bei Lenners sowohl durch das „Komm, komm“ als auch durch das unverdeckte Gesicht jene Ambiguität des Romans nicht mehr, da Esmeralda als Teufelsemissärin bzw. Femme fatale porträtiert, die Leverkühn zu ihrem Opfer macht, wird. Den darauf folgenden Geschlechtsverkehr sieht man auch grafisch kraft der plötzlichen Intervallsprünge (Abbildung 8.11):

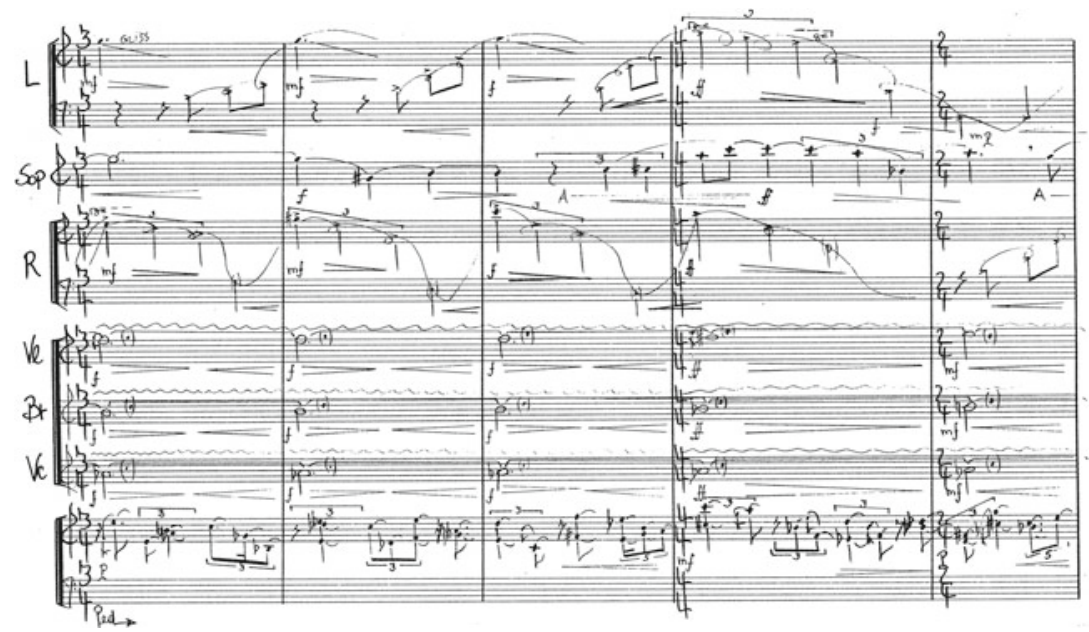

Abbildung 8.11 Die intime Beziehung (T. 192-196). Mit freundlicher Genehmigung des Autors

Die Passage, die danach vorgelesen wird, ist dem Teufelsgespräch gewidmet. Die Komposition versucht, die Kälte des Teufels mittels des Waterphones zu reproduzieren (T. 206). Das Ende dieses Abschnitts wird als „diabolico“, „teuflisch“ (T. 244) bezeichnet und von den Streichern sowie ab T. 247 auch 
vom Waterphone „più animato“, ,erregter“ gespielt. ${ }^{125}$ Bereits im vorigen Musikabschnitt wurde Esmeralda als Teufelsemissärin charakterisiert und in der Komposition hier gewinnt die Deutungsperspektive des Faust-Romans nicht nur durch die Angabe „diabolico“, sondern vor allem durch die Realisierung des Teufelsgesprächs an Bedeutung.

Dem Teufelsgespräch folgt die Echo-Episode: Die Musik soll anfänglich „leblos“ (T. 248) wirken, dann wird sie ,wehklagend, fast weinend“ (T. 265) und spielt so wörtlich und musikalisch auf die Weheklag an. Danach ist sie „energic[a]“, „kraftvoll“ (T. 275): Hier wird die feste Entscheidung Leverkühns thematisiert, die Neunte Symphonie zurückzunehmen. Das Finale des Echo-Abschnitts soll „molto calmo“, „sehr ruhig“ (T. 292) gespielt werden: Das von Leverkühn tief geliebte Kind ist gestorben.

Auf diese dramatische Episode folgt die Abschiedsrede. In T. 323 beginnt auch Esmeralda ,noch instinktiv verführerisch“ zu singen: Wie in der Oper Manzonis könnte sie hier als Projektion des wahnsinnigen Leverkühn aufgefasst werden. Dieser Eindruck wird von der Beobachtung bestätigt, dass Esmeralda nur Vokale singt. Das Motiv der Sprachkrise ist daher auch bei Lenners nicht unbedeutend: Zugleich ist die Palette der Ausdrucksmöglichkeiten der Stimme besonders groß, es herrscht ein Spannungsverhältnis zwischen Sprachkrise und Ausdruck. Ersteres lässt sich etwa an den Vokalen, die vergleichbar zu Noten fast nur auf sich selbst verweisen sowie an den kaum zu hörenden Worten der Sängerin, Letzteres am verzweifelten Schreien und den klangvollen Buchstaben festmachen. Hier findet man außerdem das anfängliche Element der Glasharfe wieder. Ab T. 335 werden der Rhythmus und die Dynamik ,più agitato“, ,erregter“ und ab T. 340 „sempre più agitato“, „,noch erregter": Der Musikverlauf der Glasharfe ist reich an Dissonanzen und der der Streicher an Trillern. Dieser Höhepunkt endet mit einer Kadenz der Glasharfe und dem Schrei der Sängerin: Der Sopran übernimmt neben der Rolle Esmeraldas auch die Rolle Leverkühns, der seine Komposition nicht aufführen kann und ohnmächtig wird. Die ,fatalen“ Auswirkungen auf ,eine in die Krise geratene Männlichkeit"126 erzählt und zeigt Esmeralda selbst. ${ }^{127}$

Ab T. 348 findet man dasselbe Musikelement wie am Anfang, sowohl im Verlauf der links positionierten Harfe als auch im Verlauf der Glasharfe: Man kann

\footnotetext{
${ }^{125}$ Die vorliegende Analyse konzentriert sich hauptsächlich auf jene Episoden, die mit der Figur Esmeralda direkt verbunden sind, und thematisiert nur kurz weitere im Stück behandelte Episoden.

${ }^{126}$ Bronfen: Liebestod und Femme fatale, S. 15.

${ }^{127}$ Vgl. DF: 729.
} 
nicht wirklich von einer Kreisstruktur sprechen, aber ohne Zweifel von einer Tendenz zur Kohäsion der verschiedenen Abschnitte. Am Ende nimmt die Sängerin, so das Manuskript, ,den Schleier ab und trägt den von Thomas Mann im Dr. Faustus erwähnten Text von Clemens Brentano vor" (S. 44). Der Anweisung kann man entnehmen, dass die Sängerin offenbar während der gesamten Aufführung einen Schleier trägt, den sie nur an exponierten Stellen abnimmt (Abbildung 8.12). $O$ lieb Mädel, wie schlecht bist du! ist ebenfalls mit dem Motiv der Versuchung durch die Frau verbunden. Wenn noch Zweifel bestanden, dass dies die Rezeptionslenkung des Stückes sei, werden diese durch die Anweisung zur Interpretation der Passage sofort aus dem Weg geräumt. Die Männerwelt wird zum Opfer der Femme fatale:

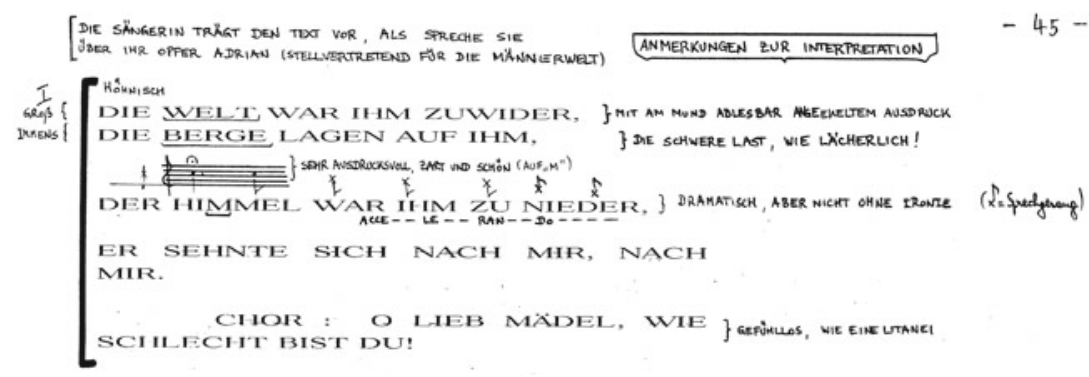

Abbildung 8.12 Die erste Strophe des Gedichts von Brentano (S. 45). Mit freundlicher Genehmigung des Autors

Esmeralda soll durch das Rezitieren in den ersten drei Strophen Ironie in der dritten singt die Sängerin beispielsweise die Melodie von Stille Nacht, was an Mahlers kompositorisches Verfahren erinnert und einen destabilisierenden Effekt erzeugt - und in der sechsten und letzten dagegen tiefen Hass ausdrücken (Abbildung 8.13).

Nicht nur kommentiert der Chor kontinuierlich, was die Sängerin sagt, auch auf der Ebene der Partitur bleibt kein Vers unkommentiert: So wie Doktor Faustus zeigt auch Lenners' Stück einen „Hang zum exzessiven Selbstkommentar“. 128 Dies erlaubt der Figur Esmeralda einerseits, von sich selbst zu erzählen, was andererseits jedoch durch die vielen Anweisungen in der Partitur eingeschränkt wird, denn die Sängerin soll beim Singen bestimmte, vorgegebene Emotionen ausdrücken.

${ }^{128}$ Börnchen: Kryptenhall, S. 68. 


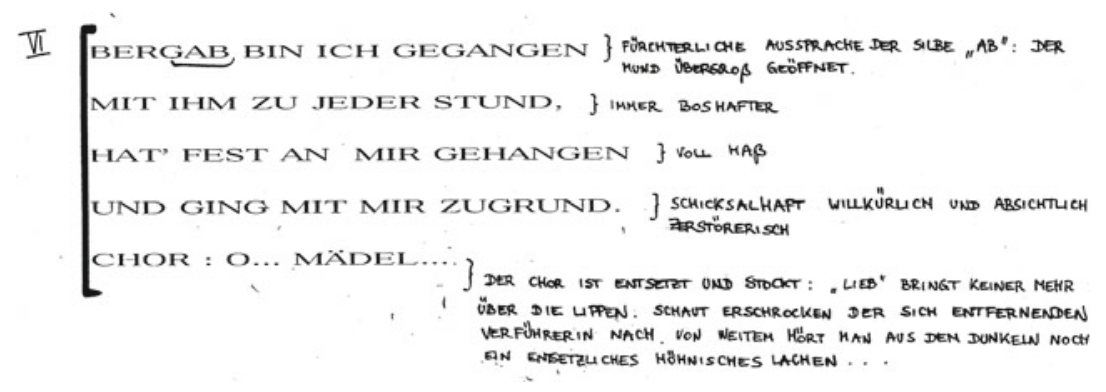

Abbildung 8.13 Die letzte Strophe (S. 46). Mit freundlicher Genehmigung des Autors

Im Roman spielt die Vertonung des Gedichts aus dem Brentano-Zyklus eine wichtige Rolle: Es ist dasjenige, in dem er ,einem strengen Satz am nächsten war“ (DF: 279) - so Leverkühn dort:

Das ist ganz aus einer Grundgestalt, einer vielfach variablen Intervallreihe, den fünf Tönen h-e-a-e-es abgeleitet, Horizontale und Vertikale sind davon bestimmt und beherrscht, soweit das eben bei einem Grundmotiv von so beschränkter Notenzahl möglich ist. Es ist wie ein Wort, ein Schlüsselwort, dessen Zeichen überall in dem Lied zu finden sind und es gänzlich determinieren möchten. (DF: 279)

Diese Komposition Leverkühns macht daher Esmeraldas Tonchiffre zu seinem kompositorischen Hauptmaterial und versucht bereits wie die Zwölftontechnik, jene Unterschiede zwischen Melodik und Harmonik zu annullieren. Der Verweis auf diese Stelle des Romans durch die Vertonung des Brentano-Gedichts, also der werkexterne Blick, widersetzt sich hier den interpretatorischen Anmerkungen und hebt doch hervor, dass die Begegnung mit Esmeralda im Leben Leverkühns nicht nur Krankheit, sondern auch „Vitaldifferenzen“129 und erneute Inspiration hervorbringt. Nur so wird das Motiv der Femme fatale, der Versuchung durch die Frau, das sonst in Lenners' Hetaera Esmeralda herrscht, in Frage gestellt.

${ }^{129}$ Freud: Jenseits des Lustprinzips, S. 55, im Original hervorgehoben. 


\subsection{4 h e a e es d und die Wahrheit der Musik: Hetaera Esmeralda in der Oper Manzonis}

In Manzonis Oper spielt Esmeralda eine wichtige Rolle, sie ist sogar wichtiger als Zeitblom. ${ }^{130}$ Die Oper beginnt mit der Bordell-Episode und das erste gesungene Wort ist: „Esmeralda!““ (M-DF: 16, T. 87-90). Außerdem wird die Tonchiffre der Prostituierten durch die gesamte Oper geführt. ${ }^{131}$ Bei Manzoni wird nicht die verkürzte Tonfolge, sondern die vollständige, h e a (e) es d, benutzt. Das erste Bild des ersten Aktes, das vom Erlebnis im Freudenhaus handelt, ist durch Farbund Lichteffekte charakterisiert: Das Bühnenbild der Uraufführungen ist anfänglich grau-grün, dann blau und am Ende der Szene rot. Musikalisch betrachtet, dominiert der Dialog zwischen Blasinstrumenten und Streichern. Die Linie der Streicher basiert hauptsächlich auf langen Tönen, die der Blasinstrumente sowie des Schlagzeugs auf schnelleren rhythmischen Figurationen.

Adrian betritt die Bühne völlig verwirrt, er weiß nicht mehr, wo er sich befindet und bewegt sich, als ob er blind wäre. Er schlägt am Klavier einige Akkorde an (M-DF: 10, T. 59 ff.), die aber wie bei Henze nicht denen des Romans entsprechen: „Das romantische ,Vokabular““, ${ }^{132}$ meint Sorg, ,ist in Manzonis Oper nicht möglich““ ${ }^{133}$ Man sieht tatsächlich kein Klavier auf der Bühne: Das Publikum ist darauf angewiesen, sich das Instrument wie etwa in den Aufführungen des elisabethanischen Theaters, wo kaum Requisiten verwendet wurden, vorzustellen. Am Ende des ersten Bildes zieht Esmeralda die Jacke aus (sie trägt also wie im Roman eine Jacke) und bewegt sich mit Sinnlichkeit, es ist aber kein echter Tanz: Manzonis Oper schreibt sich nochmals in die Tradition einer im Vergleich zur französischen Oper Tanzelemente ablehnenden Operntradition ein und orientiert

${ }^{130}$ Vgl. Manzoni: Parole per musica, S. 82. Siehe auch Wißmann, F.: Faust im Musiktheater, S. $168 \mathrm{f}$.

${ }^{131}$ Vgl. ebd. und Sorg: Beziehungszauber, S. 197.

${ }^{132}$ Sorg: Beziehungszauber, ebd. Im Roman spielt Leverkühn drei Akkorde, die an den Freischütz erinnern. Vgl. DF: 209. Das romantische Vokabular scheint aber auch in Henzes Musik trotz seines - so Manzoni bezüglich Henzes Musik - ,denso sinfonismo d'impronta romantica“ nicht möglich zu sein. „Eine reichhaltige Sinfonik romantischer Prägung“. Manzoni: Le novità della Rassegna di musica contemporanea (1959). In: Ders. (Hrsg.): Musica e progetto civile, S. 55 ff., hier: S. 56.

${ }^{133}$ Ebd. 
sich diesbezüglich ganz an dem Roman. ${ }^{134}$ Nicht nur durch die Berührung, sondern auch durch dieses performative Entkleiden versucht Esmeralda Leverkühns Aufmerksamkeit zu gewinnen.

Kurz danach beginnt das zweite Bild, das von der intimen Beziehung handelt. Die Konnotation des Ortes, die im Fall der vorher präsentierten Kompositionen eine wichtige Rolle spielte, fehlt im Gegensatz dazu bei Manzoni: Die Bühnenanweisung lautet nur ,[u]n altro luogo. Incontro con la prostituta del primo quadro“ (M-DF: 16). ${ }^{135}$

Adrian und Esmeralda singen fast die ganze Szene lang. Die im Werk des Komponisten typische Reduktion des Gesungenen auf einzelne Buchstaben ist auch hier zu sehen (Abbildung 8.14): ${ }^{136}$

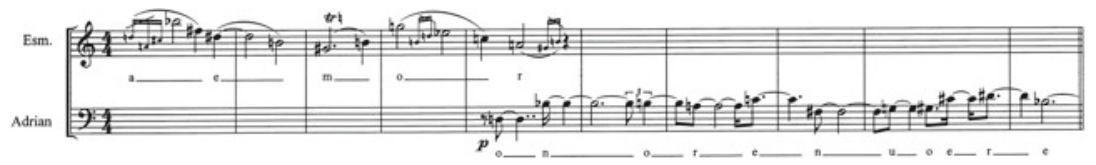

Abbildung 8.14 Adrian und Esmeralda (T. 128-137)

Adrian versucht, sich Esmeralda anzunähern, sie nimmt aber Abstand von ihm und singt entweder „Guardati dal mio corpo“ (M-DF: 18) oder „Non devi amarmi“ (M-DF: 21), also „Hüte dich vor meinem Körper“ oder „Du darfst mich nicht lieben“. ${ }^{137}$ Bei Manzoni ist es daher nicht nur der Teufel, der das Liebesverbot ausspricht: Es wird auch von Esmeralda ausgesprochen und das Motiv der Warnung kommt sowohl hier als auch an anderen Stellen der Oper deutlich zum Tragen. Somit wird betont, dass Adrian Esmeralda geliebt und sich absichtlich infiziert hat.

Kurz vor der intimen Beziehung wird das Bühnenbild feurig rot und dann nochmals grau-grün wie am Anfang des ersten Bildes: Das Ende der EsmeraldaEpisode ist damit signalisiert. Im Medium der Oper werden auf diese Weise Emotionen bzw. Affekte farblich vermittelt.

\footnotetext{
${ }^{134}$ Siehe auch Burney, Charles: The Present State of Music in France and Italy, or: The Journal of a Tour Through Those Countries, Undertaken to Collect Materials for a General History of Music. London: Becket 1773, S. 346-350.

135 „Ein anderer Ort. Begegnung mit der Prostituierten des ersten Bildes“. Zur Vagheit der Raumangaben in der Oper vgl. 5.2.1.

${ }^{136}$ Vgl. Sorg: Beziehungszauber, S. 175; Noller: Engagement und Form, S. 265. ${ }^{137}$ Vgl. DF: 225.
} 
Ein „Soprano interno“, also off-stage, singt während der Abschiedsrede ebenfalls „Guardati dal mio corpo“ (M-DF: 231; T. 230-237) und eine „donna velata“, also eine verschleierte Frau im Epilog singt ,a...r...a...i...a...1...i...o...r“ (MDF: 289. T. 715-742). ${ }^{138}$ Diese Stimmen wurden in der Premiere der Sängerin zugewiesen, die auch die Rolle der Esmeralda spielt: In den Bezeichnungen bleibt daher das Geheimnis bezüglich dieser Figur ungelöst, jedoch nicht unter gesanglich-musikalischen Gesichtspunkten, denn Esmeralda und die verschleierte Frau teilen denselben Stimmtypus. Nicht selten lässt sich bei Manzoni feststellen, dass der Vagheit in den paratextuellen Angaben eine Genauigkeit in der musikalischen Faktur entgegengesetzt wird: Die Musik umgeht ihre Selbstreferentialität und erreicht sogar eine größere Eindeutigkeit als die schriftlich fixierte Sprache. Hinter dem Schleier steht also die Wahrheit der Musik: Das metaphorisch konnotierte Motiv der Verschleierung kommt auch in der Oper vor, ebenfalls in Verbindung mit Wahrheit und Musik. Abgesehen vom Namen der Prostituierten, wird die Herkunft Esmeraldas nicht thematisiert: historisch-geographische Angaben finden in Manzonis Oper kaum Berücksichtigung; trotz der Verwendung der italienischen Sprache gewinnt Leverkühns Geschichte an Universalität, wird abstrahiert von ihrem historisch-geographischen Kontext. Zeitbloms Darstellung von Esmeralda als Femme fatale bzw. als Figur, die Leverkühn ins Unglück bringt, scheint hier keine wichtige Rolle zu spielen: Ihre Tonchiffre ist nicht unvollständig und das erste gesungene Wort lautet nicht „Hetaera Esmeralda“, was ihren Status als Prostituierte unterstrichen hätte, sondern einfach „Esmeralda“. Diese Information zu Esmeraldas sozialem Status lässt sich lediglich der Inszenierung und einigen Paratexten entnehmen: Primat hat bei Manzoni die Handlung und die fiktive Musik Leverkühns, Identitätskategorien wie Nationalität und Klasse sind zweitrangig.

Zusammenfassend ergeben sich einige Gemeinsamkeiten in der kompositorischen Rezeption der Figur Esmeralda sowie der ihr gewidmeten Kapitel, die hier, bevor aus dem gesamten Kapitel ein Fazit gezogen wird, resümiert werden sollen. Erstens übersehen sowohl Manzoni als auch Fine nicht, dass die Tonchiffre im Roman unvollständig ist und ergänzen sie in ihren Kompositionen. Im Gegensatz zu Henze, der durch den Verzicht auf die Tonchiffre ihre Bedeutung abschwächt, spielt sie jedoch bei Manzoni und Fine immerhin eine zentrale Rolle. Zweitens unterstreichen alle Kompositionen die Verbindung von Esmeralda mit der Musik, die typisch für das kulturelle Stereotyp der Femme fatale ist. Um diese Verbindung im Medium der Musik noch ausdrücklicher hervorzuheben, greifen

${ }^{138}$ Vgl. Abschn. 5.2.1.5 u. 11.2.3. 
die meisten Kompositionen auf Tanzformen wie Tango und Walzer zurück. Drittens orientieren sich die behandelten Musikwerke zum großen Teil an Zeitbloms misogyner Darstellung von Esmeralda, was bei Lenners durch die Anweisungen sowie den Rückgriff auf Brentanos Text deutlich wird.

\subsection{Fazit}

Der erste Teil des vorliegenden Kapitels konzentrierte sich auf die Darstellung der Figur Esmeralda durch Leverkühn auf der metadiegetischen Ebene der Narration und durch Zeitblom auf der intra- und extradiegetischen. Diese Charakterisierung weist viele Aspekte des kulturellen Stereotyps der Femme fatale auf und bot Anlass, über Zeitbloms nicht selten misogyne Darstellung weiblicher Figuren im Roman zu reflektieren. Die Analyse sexuell konnotierter metaphorischer Motive der Esmeralda-Kapitel, untermauert vom intramedialen Verweis auf Freuds Schrift Jenseits des Lustprinzips, brachte nicht nur die sexualisierte Charakterisierung der Prostituierten ans Licht, sondern auch von vielen weiblichen Figuren von Doktor Faustus. Viele dieser Motive - die Nacktheit, die Durchsichtigkeit, der Durchbruch - tauchen auch in Verbindung mit den Topoi der Sterilität der Kunst und der (nackten) Wahrheit des Textes und der Musik auf. Die in diesem Kapitel behandelten Kompositionen unterstützen oft die Zuordnung Esmeraldas zum kulturellen Stereotyp der Femme fatale, indem sie auf Tanzformen zurückgreifen (den Walzer bei Fine, den Tango bei Henze) oder das Motiv der Versuchung durch die Frau ins Zentrum rücken (Lenners). Dass die Tonchiffre der Prostituierten im Roman unvollständig ist, was ihre misogyne Darstellung noch deutlicher hervorhebt, übersehen die Kompositionen von Fine und Manzoni jedoch nicht und ergänzen sie um die Note d. Die Analyse zweier verdeckter intermedialer Produkte (Fine und Henze) bot außerdem die Möglichkeit, über Grenzen und Potenziale der Figurencharakterisierung im Medium der instrumentalen Musik nachzudenken. Beispielsweise ermöglicht die Vagheit, die diesbezüglich auffällig wird, keine klare Darstellung wie im Medium des Romans. Somit wird jedoch sowohl die Vorstellungskraft von Rezipient*innen angeregt als auch die emotionale Welt der Figur effektiver gezeigt bzw. hörbar gemacht. In Hinblick auf die Forschungsfragen der Arbeit lässt sich demnach Folgendes sagen: Indem dieses Kapitel noch ein Stück kompositorischer Rezeptionsgeschichte von Doktor Faustus beleuchtet hat, ist es auch im Prozess des Medienvergleichs der Frage nach den Möglichkeiten der Figurencharakterisierung sowohl im Medium der Literatur als auch der Musik, selbst in dem der instrumentalen Musik, nachgegangen. 
Im nächsten Kapitel wird sich die intermedial angelegte Untersuchung erneut mit einer Figur aus Thomas Manns Roman befassen, und zwar mit dem Geiger Rudolf Schwerdtfeger. Dabei wird nochmals über die Darstellung einer Musikerfigur in der Literatur und in der Musik reflektiert. Anhand des Konzerts von Hans Werner Henze soll außerdem die in diesem Kapitel bereits angerissene Frage nach den Möglichkeiten der Figurencharakterisierung im Medium der instrumentalen Musik vertieft werden.

Open Access Dieses Kapitel wird unter der Creative Commons Namensnennung - Nicht kommerziell - Keine Bearbeitung 4.0 International Lizenz (http://creativecommons.org/lic enses/by-nc-nd/4.0/deed.de) veröffentlicht, welche die nicht-kommerzielle Nutzung, Vervielfältigung, Verbreitung und Wiedergabe in jeglichem Medium und Format erlaubt, sofern Sie den/die ursprünglichen Autor(en) und die Quelle ordnungsgemäß nennen, einen Link zur Creative Commons Lizenz beifügen und angeben, ob Änderungen vorgenommen wurden. Die Lizenz gibt Ihnen nicht das Recht, bearbeitete oder sonst wie umgestaltete Fassungen dieses Werkes zu verbreiten oder öffentlich wiederzugeben.

Die in diesem Kapitel enthaltenen Bilder und sonstiges Drittmaterial unterliegen ebenfalls der genannten Creative Commons Lizenz, sofern sich aus der Abbildungslegende nichts anderes ergibt. Sofern das betreffende Material nicht unter der genannten Creative Commons Lizenz steht und die betreffende Handlung nicht nach gesetzlichen Vorschriften erlaubt ist, ist auch für die oben aufgeführten nicht-kommerziellen Weiterverwendungen des Materials die Einwilligung des jeweiligen Rechteinhabers einzuholen.

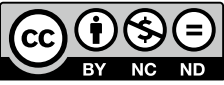

